\title{
Preparation Methods for Graphene Metal and Polymer Based Composites for EMI Shielding Materials: State of the Art Review of the Conventional and Machine Learning Methods
}

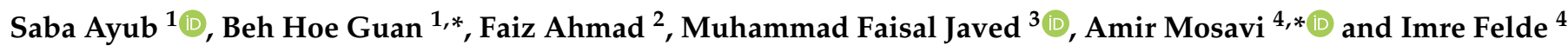 \\ 1 Department of Fundamental and Applied Sciences, Universiti Teknologi PETRONAS, \\ Bandar Seri Iskandar 32610, Malaysia; saba_20000009@utp.edu.my \\ 2 Department of Mechanical Engineering, Universiti Teknologi PETRONAS, \\ Bandar Seri Iskandar 32610, Malaysia; faizahmad@utp.edu.my \\ 3 Department of Civil Engineering, COMSATS University Islamabad Abbottabad Campus, \\ Abbottabad 22060, Pakistan; arbabfaisal@cuiatd.edu.pk \\ 4 John von Neumann Faculty of Informatics, Obuda University, 1034 Budapest, Hungary; \\ felde.imre@uni-obuda.hu \\ * Correspondence: beh.hoeguan@utp.edu.my (B.H.G.); amir.mosavi@uni-obuda.hu (A.M.)
}

\section{check for}

updates

Citation: Ayub, S.; Guan, B.H.; Ahmad, F.; Javed, M.F.; Mosavi, A.; Felde, I. Preparation Methods for Graphene Metal and Polymer Based Composites for EMI Shielding Materials: State of the Art Review of the Conventional and Machine Learning Methods. Metals 2021, 11, 1164. https://doi.org/10.3390/ met11081164

Academic Editor: Jacek Trzaska

Received: 10 June 2021

Accepted: 17 July 2021

Published: 22 July 2021

Publisher's Note: MDPI stays neutral with regard to jurisdictional claims in published maps and institutional affiliations.

Copyright: (c) 2021 by the authors. Licensee MDPI, Basel, Switzerland. This article is an open access article distributed under the terms and conditions of the Creative Commons Attribution (CC BY) license (https:/ / creativecommons.org/licenses/by/ $4.0 /)$.
Abstract: Advancement of novel electromagnetic inference (EMI) materials is essential in various industries. The purpose of this study is to present a state-of-the-art review on the methods used in the formation of graphene-, metal- and polymer-based composite EMI materials. The study indicates that in graphene- and metal-based composites, the utilization of alternating deposition method provides the highest shielding effectiveness. However, in polymer-based composite, the utilization of chemical vapor deposition method showed the highest shielding effectiveness. Furthermore, this review reveals that there is a gap in the literature in terms of the application of artificial intelligence and machine learning methods. The results further reveal that within the past half-decade machine learning methods, including artificial neural networks, have brought significant improvement for modelling EMI materials. We identified a research trend in the direction of using advanced forms of machine learning for comparative analysis, research and development employing hybrid and ensemble machine learning methods to deliver higher performance.

Keywords: electromagnetic inferences; shielding; graphene; metal; polymer; traditional methods; machine learning; artificial intelligence; data science; materials design

\section{Introduction}

Electromagnetic pollution is rapidly increasing which not only affects electronic equipment but is also harmful to the environment, ecosystem, and the public health [1]. Electromagnetic waves damage human health in various forms such as psychological disorders, affecting the immune system and also causing problems in hereditary scenarios, and with time their impact is increasing which requires vital attention [2]. Research on electromagnetic shielding has emerged as early as 1830s by evolving the Faraday's cage, i.e., an encircling conductive housing shield with zero electric fields [3]. Therefore, there is a need for appropriate materials that acts as shields to counter electromagnetic waves [4]. Electromagnetic shielding requires a balanced combination between electrical conductivity, dielectric permittivity, and magnetic permeability. It is also observed that the material' morphology and aspect ratio play an important role in electromagnetic shielding and the factors introduced are reflection, absorption, and multiple reflection losses [5-8].

In a material, the main mechanism for electromagnetic interference attenuation are absorption, reflection, and multiple reflections [9]. Reflection is a primary shielding mechanism that occurs in highly electrically conductive structures such as metals. The reflection phenomena depend on mobile charge carriers such as electrons which are present within 
the material. Therefore, the shielding material is likely to be electrically conductive although it is not an essential requirement [10]. The second mechanism for electromagnetic shielding is absorption [11]. It requires the existence of electric and magnetic dipoles to interact with the electromagnetic radiations and greatly depends on the thickness. The third mechanism of electromagnetic shielding is multiple reflections which require large surface areas to interfaces within the shield [12]. The four most common methods used for the measurement of electromagnetic shielding are (1) open field or free space method, (2) shield box method, (3) shield room method, and (4) coaxial transmission line method [13]. Over the past decade, metals were commonly used materials to overcome the electromagnetic shielding interference issue due to their good electrical conductivity and overall shielding effectiveness; however, metals have many disadvantages such as high mass density, corrosion and difficult processing [14,15]. In order to achieve good shielding effectiveness, many other materials are introduced such as carbon, graphene and conducting polymers [15]. Graphene, although it is non-metal, exhibits properties similar to semi-conducting metals which makes it suitable for electromagnetic inferences (EMI) applications [16-19]. However, conducting polymers have problems of poor mechanical strength and low processability. The proper distribution of carbon-based filler material within the polymer matrix can be effective to obtain good electromagnetic shielding effectiveness, where polymer-based composite materials improve the absorption and as well reflecting incoming radiation $[20,21]$.

The fabrication of materials can be accomplished by using different methods where researchers try different methods to build a new composite. The selection of methods varies from material to material, for example, for the fabrication of metals, friction stir processing and stir casting are mostly used [22]. Similarly, for other materials researchers used different methods according to the properties of materials that are suitable for the preparation of new composites [23]. The selection of an appropriate method plays a vital role in achieving EMI shielding effectiveness by forming a homogenous sample. Besides these methods, the internal properties of materials also have a significant impact on electromagnetic shielding effectiveness. Various studies have been conducted on different types of available methods to obtain the maximum EMI shielding of a composite. This review aims to assess the various traditional and artificial intelligence methods to synthesize the shielding composites to deal with EMI. This paper builds on a previous review [24] conducted on the applications of graphene, iron and polymer composites in EMI shielding, where the top materials were highlighted in each frequency range to secure good shielding effectiveness. In this review preparation methods that help to build the EMI shielding composites have been reviewed. The method selection affects the properties of the material hence impacting the EMI shielding. The scope of this study is limited to a review the research articles of graphene- and metal-based composites, and graphene-, metal- and polymer-based composites formulated through various methods (traditional and artificial intelligence). Although there are many types of carbon materials, not all are suitable for EMI applications. Graphene is an emerging material that shows remarkable results as a composite in EMI applications, which is why it was chosen over other types. Another reason for focusing on these materials and their combination is that graphene-, metal- and polymer-based composites show good performance in EMI applications, therefore reviewing their methods is more pertinent. This study sets a benchmark for future researchers to select the most appropriate method in a selected composite family to formulate a new shielding material.

\section{Review Methodology}

The methodology of this review exhibits the extraction of those articles which were published on the composite formation via traditional methods of various materials as electromagnetic shielding materials. Popular materials such as carbon, graphene, iron and polymer were taken into consideration, as it is important to know about their manufacturing behavior which impacts significantly on shielding effectiveness. VOSviewer software (version 1.6.11, 2021, Centre for Science and Technology Studies, Leiden University, 
Leiden, The Netherlands) was used to make keyword analysis of graphene- and metalbased composites, and graphene-, metal- and polymer-based composites articles. Furthermore, EMI studies related to artificial intelligence were also reviewed. A summary of the review methodology is demonstrated in Figure 1.

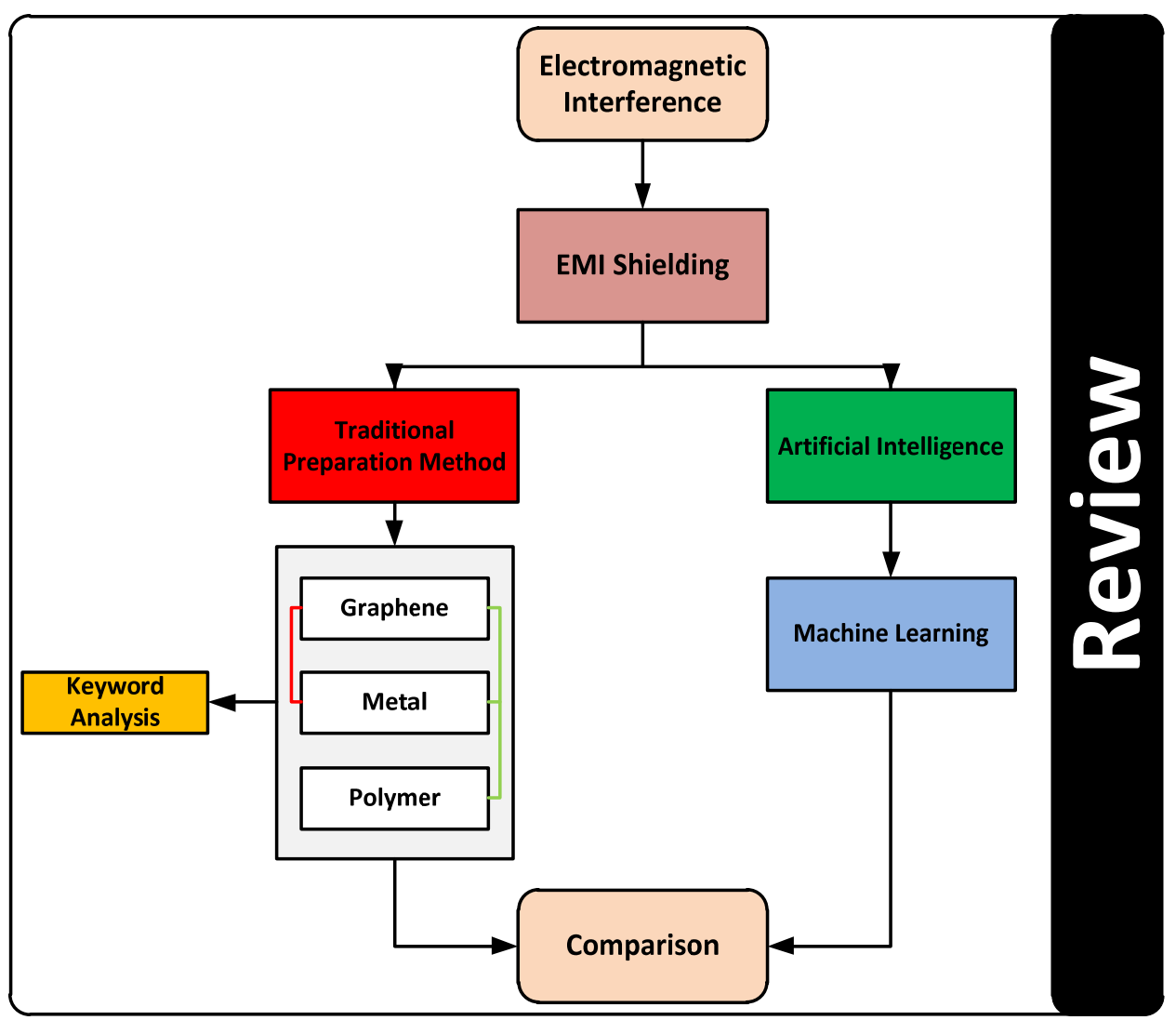

Figure 1. Review flowchart.

\section{Interpretation of Articles}

This section covers the summary of the extracted articles (traditional methods) which were published on the preparation methods for graphene-, metal- and polymer-based composites for EMI shielding materials. Besides that, VOSviewer software was used to show the mapping of the extracted articles based on the keywords co-occurrence.

\subsection{Summary of Extracted Articles}

The English language articles were extracted using the Google Scholar search engine without applying any year limitations. The reason we used Google Scholar for article extraction was that it covers published work from all journals, either from the Web of Science index, Scopus index or anywhere else. Figure 2 shows the distribution of the articles of graphene- and metal-based composites, and Figure 3 shows the distribution of the articles of polymer-based composites. The number of publications is limited as only those articles which come under the formed combination, i.e., graphene- and metal-based composites, and graphene-, metal- and polymer-based composites were taken into account. A gradual increase in the publications occurred over time. An interesting thing which was also observed that with time the focus is more towards the polymer-based composites as they emerged as promising shielding materials. 


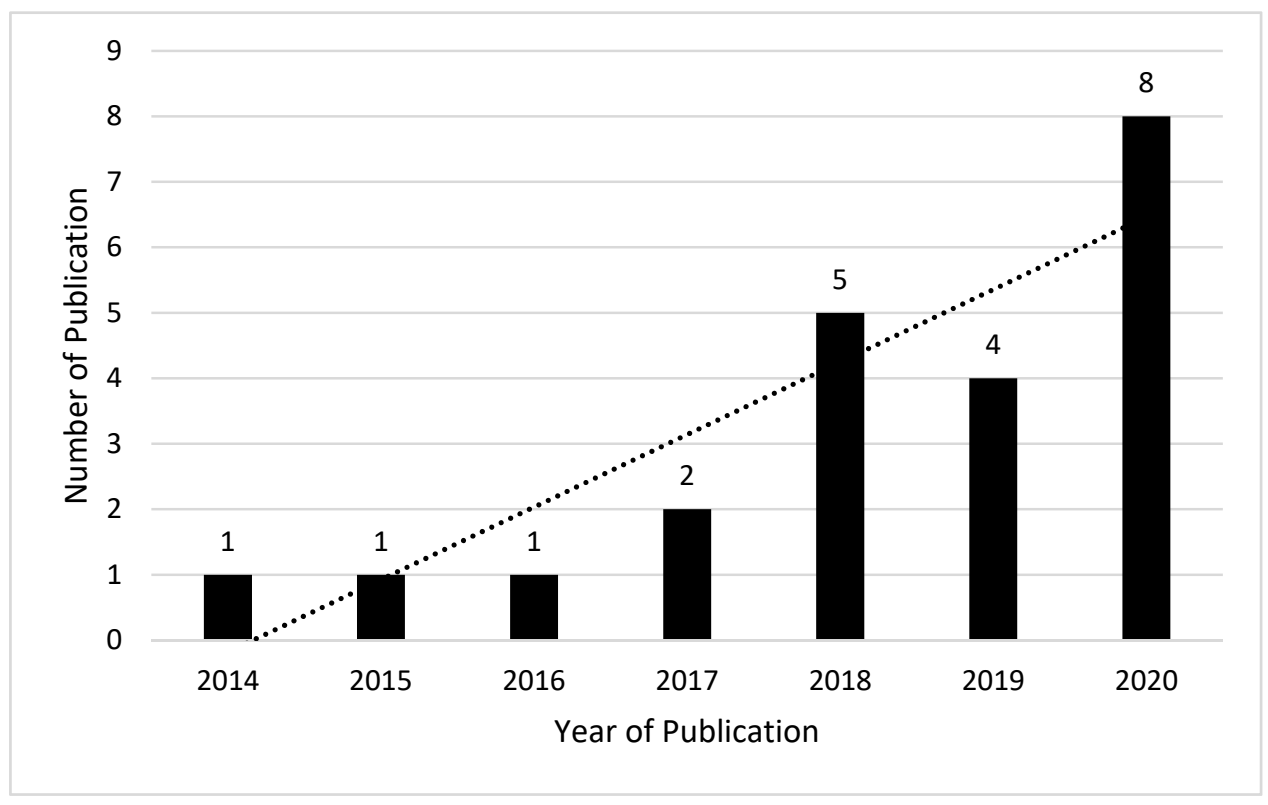

Figure 2. Articles distribution of graphene- and metal-based composites.

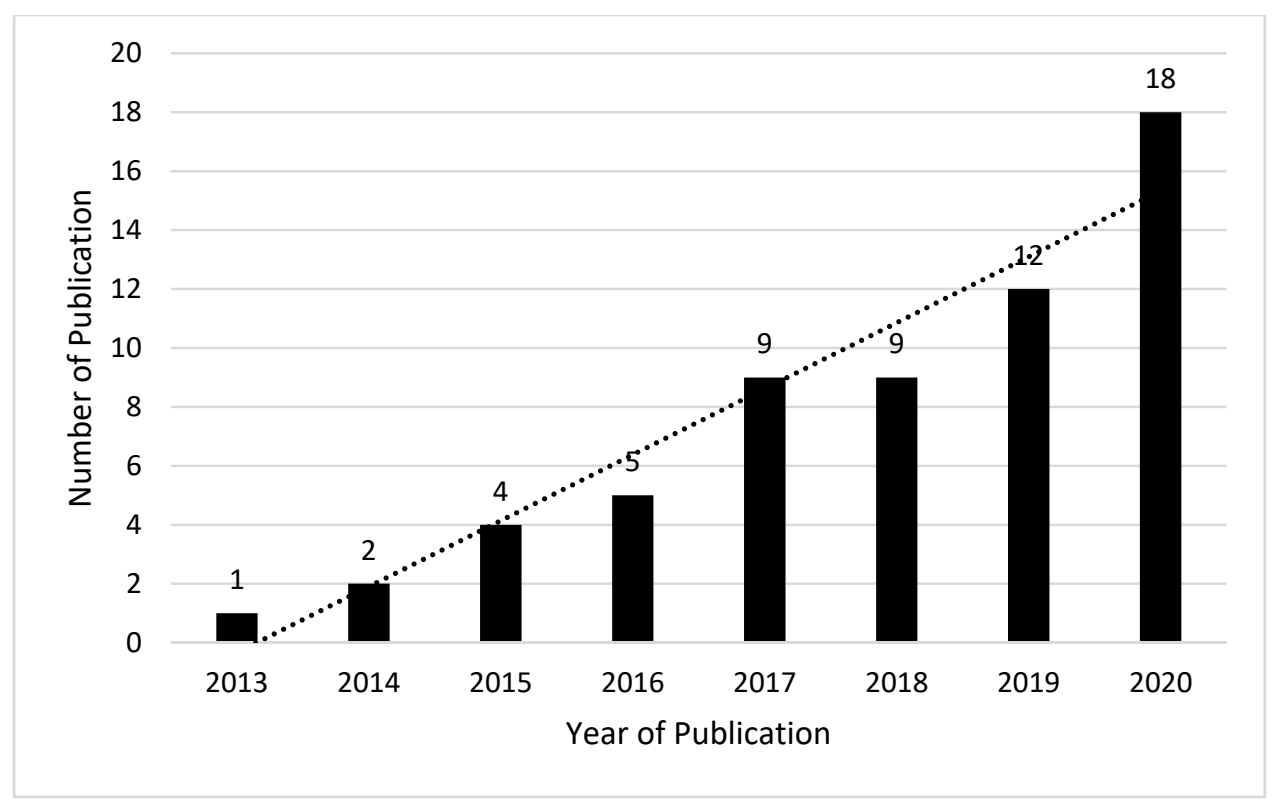

Figure 3. Articles distribution of polymer-based composites.

\subsection{Keywords Analysis}

Keywords are an important component of a research article that provide useful information on a paper as well as an area of interest. A comprehensive analysis of keywords in various technical fields can help demonstrate trends in research growth and differences. In many papers, co-occurrence analysis of keywords was often used to determine the extent of the relations between different keywords. The link and role of internal materials can be best grasped up in an academic domain by researching keyword co-occurrence relationships and revealing the research limits of the discipline. In the current analysis, a linking of the details based on keywords from the selected papers was generated with the help of VOSviewer software as seen in Figure 4. 


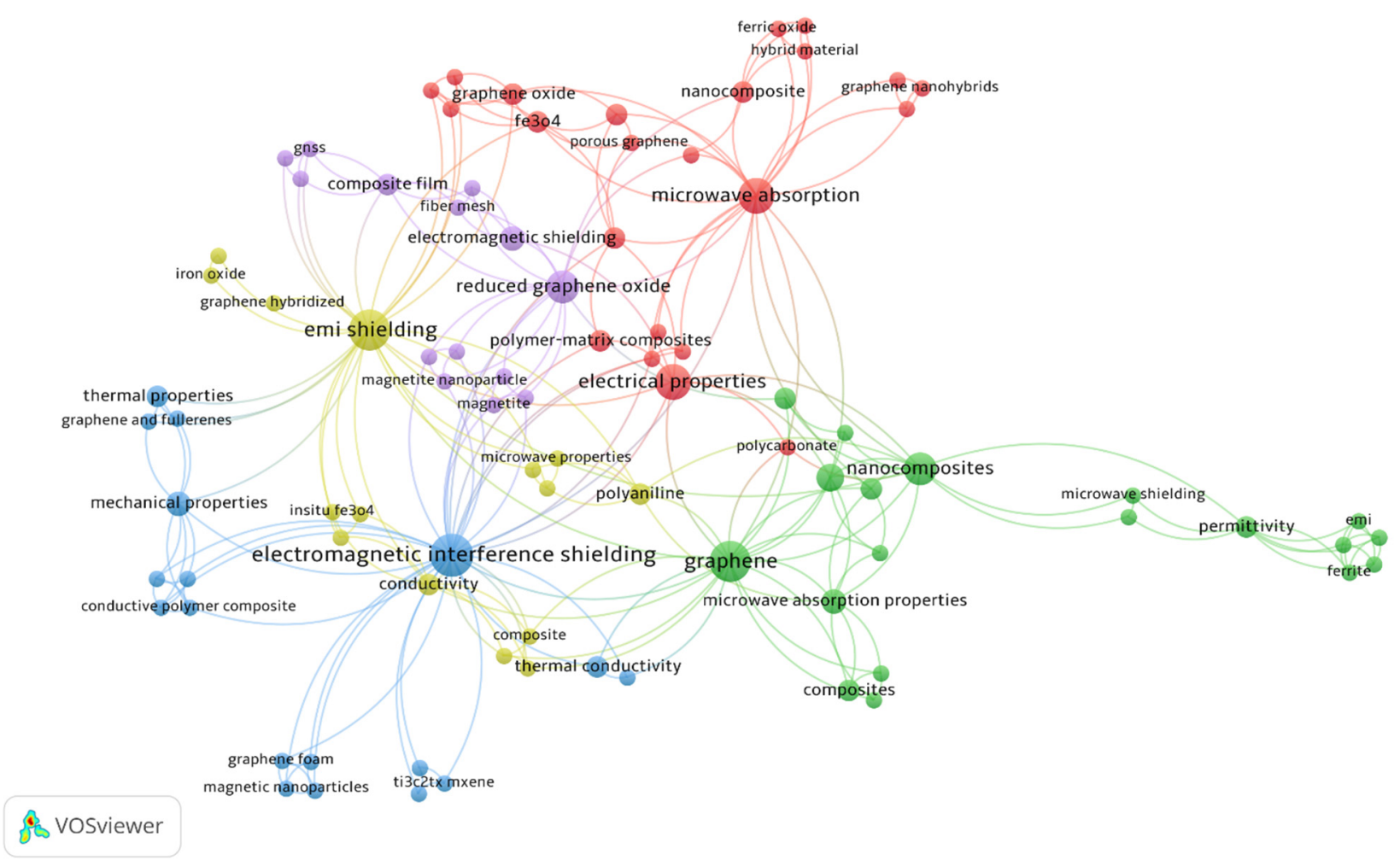

Figure 4. Mapping of co-occurrence Keywords.

Keyword occurrence was analyzed using VOSviewer's "full counting" technologies and a minimal number of keywords occurrence was set to 1 . A total of 89 eligible keywords were defined by the software that reaches the threshold. The mapping network of 89 linked recurrent keywords with five fuzzy clusters was developed by setting the cluster limit at least 13 cluster keywords. The cluster nodes represent a keyword that associates the connection with other nodes.

Blue nodes with 13 occurrences, which is the first cluster, were built on the terminology "Electromagnetic interference shielding". In the same color pattern, the terms "Mechanical properties", "Thermal properties" and "Thermal conductivity" with occurrences three and two, respectively, can also be seen. The following cluster also includes some other keywords and the linkage between all the keywords shows the relation of each keyword in a particular domain. Green nodes with 11 occurrences, which is the second cluster, were built on the terminology "Graphene". In the same color pattern, a few keywords like "nanocomposites", having six occurrences, and "Microwave absorption properties" with three occurrences, were also presented. Other keywords such as "absorption properties", "magnetic property" and "permeability" show researchers' interest in this region. Yellow nodes with 11 occurrences, which is the third cluster are built on the terminology "EMI shielding". This cluster is augmented with various polymers keywords like "Single wall carbon nanohorn, "Insitu $\mathrm{Fe}_{3} \mathrm{O}_{4}$ " and "Iron Oxide". The fourth prominent cluster had red nodes around the term "Microwave absorption" and "Electrical properties" with eight occurrences both. The fifth prominent cluster had green nodes having the keywords "Reduced graphene oxide", with six occurrences.

\section{Discussion of Articles}

This section covers the compilation of the various methods used for the formulation of carbon, metals, graphene, iron, and polymer family materials. Based on the methods, a discussion has been provided which identify the most suitable methods in each family. The overall summary of the available traditional methods is provided in Table 1. 
Table 1. Various mixing methods.

\begin{tabular}{|c|c|c|c|c|c|}
\hline S. No & Method & Reference & Remarks & Advantage & Disadvantage \\
\hline 1 & $\begin{array}{l}\text { Chemical vapor } \\
\text { deposition }\end{array}$ & [25-27] & $\begin{array}{l}\text { A deposition process performed at } \\
\text { high temperature and gas pressure } \\
\text { and provides better optical and } \\
\text { electrical properties in } \\
\text { graphene-based composites. }\end{array}$ & $\begin{array}{ll}\text { - } & \text { Recommended for } \\
\text { - } & \text { Goating } \\
& \text { Gives high dispersion }\end{array}$ & $\begin{array}{l}\text { Not suitable for } \\
\text { organic materials }\end{array}$ \\
\hline 2 & $\begin{array}{l}\text { Alternating } \\
\text { deposition }\end{array}$ & [28] & \multicolumn{3}{|c|}{ Need further exploration. } \\
\hline 3 & $\begin{array}{l}\text { Electrophoretic } \\
\text { deposition }\end{array}$ & [29] & $\begin{array}{l}\text { Most used process for } \\
\text { material coating }\end{array}$ & $\begin{array}{ll}- & \text { Easy to use } \\
\text { - } & \text { The deposition rate } \\
& \text { is high } \\
\text { - } & \text { Binder elimination } \\
\text { - } & \text { Can adopt any shape }\end{array}$ & - $\quad$ Limited adhesion \\
\hline 4 & In situ growth & [30-41] & $\begin{array}{l}\text { This technique is a novel way to } \\
\text { implant graphene layers on metal } \\
\text { without any damage to graphene. } \\
\text { However, structural control by } \\
\text { this technique needs } \\
\text { further investigation. }\end{array}$ & $\begin{array}{ll}\text { - } & \text { Wrinkle-free } \\
& \text { High-quality } \\
\text { dispersion } \\
\text { - } & \text { Lithography-free } \\
& \end{array}$ & $\begin{array}{ll}-\quad & \text { Expensive procedure } \\
-\quad & \text { Time consuming }\end{array}$ \\
\hline 5 & $\begin{array}{l}\text { Thermal } \\
\text { annealing } \\
\text { method }\end{array}$ & {$[42,43]$} & $\begin{array}{l}\text { This thermal annealing method } \\
\text { used to modify the surface } \\
\text { morphology of materials with } \\
\text { temperature and time. It is a mostly } \\
\text { useable method for intrinsic, } \\
\text { structure improving and surface } \\
\text { roughness control in materials and } \\
\text { is well used for stress liberation. }\end{array}$ & $\begin{array}{ll}\text { - } & \text { Improve structure } \\
\text { Eliminate surface } \\
\text { roughness }\end{array}$ & - $\quad$ Time consuming \\
\hline 6 & $\begin{array}{l}\text { Facile synthetic } \\
\text { route }\end{array}$ & [44-47] & $\begin{array}{l}\text { Mostly a commonly used method to } \\
\text { synthesize porous structures. }\end{array}$ & $\begin{array}{ll}\text { - } & \text { Cheap process } \\
\text { - } & \text { Environment friendly }\end{array}$ & $\begin{array}{l}\text { - Nanoparticles } \\
\text { formation is slow }\end{array}$ \\
\hline 7 & $\begin{array}{l}\text { Hydrothermal } \\
\text { method }\end{array}$ & {$[41,48-56]$} & $\begin{array}{l}\text { Involves substance crystallization at } \\
\text { high temperature and pressure. }\end{array}$ & 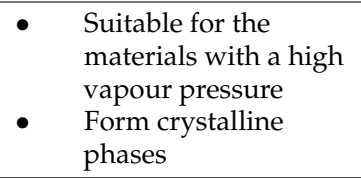 & $\begin{array}{ll}\text { - } & \text { No access to reaction } \\
\text { process } \\
\text { Expensive autoclave } \\
\text { required }\end{array}$ \\
\hline 8 & Scalable method & [57] & \multicolumn{3}{|c|}{ Need further exploration. } \\
\hline 9 & $\begin{array}{l}\text { Solvothermal } \\
\text { method }\end{array}$ & [58-63] & $\begin{array}{l}\text { This technique is used to form a } \\
\text { chemical composite. The benefit of } \\
\text { using this technique is that it } \\
\text { involves the usage of sol-gel and } \\
\text { hydrothermal routes, providing } \\
\text { precise control over the shape, size } \\
\text { and crystallinity of composites. }\end{array}$ & $\begin{array}{l}\text { - Suitable for all types } \\
\text { of materials } \\
\text { Good control over the } \\
\text { size and distribution } \\
\text { of the material }\end{array}$ & $\begin{array}{ll}\text { - } & \text { No access to reaction } \\
\text { process } \\
\text { - } \\
\text { Expensive autoclave } \\
\text { required }\end{array}$ \\
\hline
\end{tabular}

\begin{tabular}{|c|c|c|c|c|c|}
\hline 10 & $\begin{array}{l}\text { Filtration- } \\
\text { assisted } \\
\text { self-assembly } \\
\text { method }\end{array}$ & [64] & & eed further exploration. & \\
\hline 11 & $\begin{array}{l}\text { Wet stirring } \\
\text { process }\end{array}$ & [65] & $\begin{array}{l}\text { A simple technique of stirring } \\
\text { which deals with homogenous } \\
\text { mixing of liquids and stir up the } \\
\text { solid particle into liquid by using } \\
\text { water as a solvent. }\end{array}$ & $\begin{array}{ll}- & \text { Easy to use } \\
- & \text { Cost efficient }\end{array}$ & $\begin{array}{l}\text { - A high amount of } \\
\text { diluent is required }\end{array}$ \\
\hline 12 & $\begin{array}{l}\text { Self-assembly } \\
\text { technique }\end{array}$ & {$[54,66]$} & $\begin{array}{l}\text { In this method without using any } \\
\text { external direction among } \\
\text { components the disordered system } \\
\text { and pre-existing components make } \\
\text { it to an organized structure or } \\
\text { pattern, it is a low-cost approach } \\
\text { for nanofabrication. }\end{array}$ & $\begin{array}{ll}\text { - } & \text { Cheap process } \\
\text { - } & \text { Organized process }\end{array}$ & $\begin{array}{ll}- & \text { Time consuming } \\
\text { - } & \text { High cost }\end{array}$ \\
\hline
\end{tabular}


Table 1. Cont.

\begin{tabular}{|c|c|c|c|c|c|}
\hline S. No & Method & Reference & Remarks & Advantage & Disadvantage \\
\hline 13 & $\begin{array}{l}\text { Vacuum-assisted } \\
\text { filtration method }\end{array}$ & [67] & & eed further exploration. & \\
\hline 14 & $\begin{array}{l}\text { Solution } \\
\text { processing } \\
\text { method }\end{array}$ & [68-77] & $\begin{array}{l}\text { A promising method to produce } \\
\text { low-cost composites. This method is } \\
\text { used mostly in organic materials } \\
\text { such as polymer-based composites. } \\
\text { It is used in different ways through } \\
\text { high-speed shear mixing, } \\
\text { ultrasonication and as well stirring } \\
\text { for the formation of polymer } \\
\text { nanocomposites where the mixing } \\
\text { depends on the solvent. A good } \\
\text { dispersion of carbon nanofiller in } \\
\text { polymer matrixes can also } \\
\text { be achieved. }\end{array}$ & - Less expensive & $\begin{array}{l}\text { - Difficult coating } \\
\text { process }\end{array}$ \\
\hline 15 & $\begin{array}{l}\text { Chemical } \\
\text { oxidative } \\
\text { polymerization }\end{array}$ & [78-81] & $\begin{array}{l}\text { In this method, oxidizing agents } \\
\text { are used to forming a } \\
\text { polymer-based composite. }\end{array}$ & $\begin{array}{l}\text { - Suitable for polymer } \\
\text { synthesis }\end{array}$ & - $\quad$ Slow polymerization \\
\hline 16 & $\begin{array}{l}\text { Co-precipitation } \\
\text { method }\end{array}$ & {$[82,83]$} & $\begin{array}{l}\text { Used to synthesized iron } \\
\text { nanoparticles. }\end{array}$ & $\begin{array}{ll}- & \text { Simple process } \\
- & \text { Low cost } \\
\end{array}$ & $\begin{array}{l}\text { - } \quad \text { Poor control on the } \\
\text { particle size } \\
\text { distribution }\end{array}$ \\
\hline 17 & $\begin{array}{c}\text { Centrifugal } \\
\text { mixing method }\end{array}$ & [84] & \multicolumn{3}{|c|}{ Need further exploration. } \\
\hline 18 & $\begin{array}{l}\text { Citrate precursor } \\
\text { method }\end{array}$ & [85] & $\begin{array}{l}\text { A chelate-based method that is } \\
\text { efficient to reduce the metal ions for } \\
\text { nanoparticles fabrication and also } \\
\text { stimulate reaction conditions. }\end{array}$ & $\begin{array}{ll}\text { - } & \text { Low cost } \\
\text { - } & \text { Less cracking in } \\
\text { composite due to } \\
\text { internal heat } \\
\text { distribution } \\
\text { - Good atomic-level } \\
\text { mixing }\end{array}$ & $\begin{array}{l}\text { - Difficult to control the } \\
\text { parameters }\end{array}$ \\
\hline 19 & $\begin{array}{l}\text { Chemical } \\
\text { reduction }\end{array}$ & [15] & $\begin{array}{l}\text { A cost-effective method and widely } \\
\text { used in for mass production of } \\
\text { reduced graphene oxide in which } \\
\text { reduction agent used in the form of } \\
\text { gas or liquid in graphene oxide for } \\
\text { the elimination of functional group. }\end{array}$ & $\begin{array}{l}\text { - } \quad \text { Mass production } \\
\text { - Cost effective }\end{array}$ & $\begin{array}{l}\text { - Not suitable for all } \\
\text { composites. }\end{array}$ \\
\hline 20 & $\begin{array}{l}\text { Hot-molding } \\
\text { process }\end{array}$ & [86] & $\begin{array}{l}\text { This process is useful for adding } \\
\text { thermoplastic binders to the } \\
\text { metallic and ceramic powder to } \\
\text { make it fluent. In all this process } \\
\text { temperature used above then the } \\
\text { melting point. }\end{array}$ & $\begin{array}{l}\text { Effective for the lesser } \\
\text { amount of materials } \\
\text { - Cheap process }\end{array}$ & $\begin{array}{l}\text { - } \quad \text { Contamination risk } \\
\text { - } \quad \text { Slow process }\end{array}$ \\
\hline 21 & $\begin{array}{l}\text { Mechanical } \\
\text { mixing }\end{array}$ & [87] & $\begin{array}{l}\text { Used to form a uniform coat of the } \\
\text { particles on the material surface } \\
\text { where pellets are followed by a } \\
\text { cooling process. }\end{array}$ & $\begin{array}{l}\text { - Cheap and easy } \\
\text { process }\end{array}$ & $\begin{array}{l}\text { - High share force not } \\
\text { suitable for graphene } \\
\text { composite }\end{array}$ \\
\hline 22 & $\begin{array}{c}\text { Dilute } \\
\text { polymerization }\end{array}$ & [88] & \multicolumn{3}{|c|}{ Need further exploration. } \\
\hline 23 & $\begin{array}{l}\text { High-pressure } \\
\text { solid-phase } \\
\text { compression } \\
\text { molding }\end{array}$ & [88] & $\begin{array}{l}\text { An old material processing } \\
\text { technique. In industrial methods } \\
\text { which are used for plastic, it was a } \\
\text { commonly used method. }\end{array}$ & $\begin{array}{ll}\text { - } & \text { Effective for } \\
\text { thermoplastic } \\
\text { composite } \\
\text { - } \quad \text { Cheap process }\end{array}$ & $\begin{array}{l}\text { - Not recommended on } \\
\text { high-scale mass } \\
\text { production }\end{array}$ \\
\hline 24 & $\begin{array}{l}\text { Injection } \\
\text { molding process }\end{array}$ & [89] & $\begin{array}{l}\text { A high volume and low-pressure } \\
\text { process which is performed with } \\
\text { filled thermoplastics. }\end{array}$ & $\begin{array}{ll}- & \text { High production rate } \\
- & \text { Easy to mold } \\
\text { - } & \text { Suitable for most of } \\
& \text { the materials }\end{array}$ & $\begin{array}{l}\text { Costly for the lesser } \\
\text { amount of materials }\end{array}$ \\
\hline
\end{tabular}


Table 1. Cont.

\begin{tabular}{|c|c|c|c|c|c|}
\hline S. No & Method & Reference & Remarks & Advantage & Disadvantage \\
\hline 25 & $\begin{array}{l}\text { Ultrasonication } \\
\text { technique }\end{array}$ & [90-92] & $\begin{array}{l}\text { A technique used for the } \\
\text { preparation of nanoparticles. It has } \\
\text { good control over the structure of } \\
\text { the material. Moreover, with this } \\
\text { technique the size of a previously } \\
\text { formed composite can also } \\
\text { be reduced. }\end{array}$ & $\begin{array}{l}\text { - Controlled structure } \\
\text { of materials }\end{array}$ & $\begin{array}{l}\text { - } \quad \text { High energy } \\
\text { consumption }\end{array}$ \\
\hline 26 & $\begin{array}{l}\text { Hummer's } \\
\text { method }\end{array}$ & [93-97] & $\begin{array}{l}\text { A chemical process mostly used to } \\
\text { produce graphene from graphite. }\end{array}$ & $\begin{array}{ll}\text { - } & \text { Cheap process } \\
\text { - } & \text { Time saving } \\
\text { - } & \text { Highly efficient }\end{array}$ & $\begin{array}{ll} & \text { Release toxic gases } \\
\text { during } \\
\text { experimentation }\end{array}$ \\
\hline 27 & $\begin{array}{l}\text { Hot compressed } \\
\text { method }\end{array}$ & {$[14,98-101]$} & $\begin{array}{l}\text { The hot compression method cannot } \\
\text { work at room temperature like the } \\
\text { cold compression method as it takes } \\
\text { place by applying heat to the mold. }\end{array}$ & $\begin{array}{l}\text { - } \quad \text { Preferable for smaller } \\
\text { production } \\
\text { - Cheap process }\end{array}$ & $\begin{array}{ll}- & \text { Slow process } \\
- & \text { Damaging to molds } \\
- & \text { Contamination risk }\end{array}$ \\
\hline 28 & $\begin{array}{l}\text { 3D printing } \\
\text { method }\end{array}$ & [102] & $\begin{array}{l}\text { A new method to form a shielding } \\
\text { material followed by Object's } \\
\text { PolyJet Matric printing technology, } \\
\text { where a couple of materials are } \\
\text { built simultaneously. }\end{array}$ & $\begin{array}{l}\text { - } \quad \text { Time efficient } \\
\text { Less parameters } \\
\text { involved }\end{array}$ & $\begin{array}{ll}-\quad & \text { No mass production } \\
\text { - } & \text { High cost }\end{array}$ \\
\hline
\end{tabular}

\subsection{Traditional Methods}

\subsubsection{Methods for Preparation of Graphene- and Metal-Based Composites}

Over the years, graphene has gained attention in the field of research due to its tremendous properties. Graphene is wrapped in the honeycomb crystal lattice and is a one-atom-thick planar sheet [103]. Graphene possesses optical transparency, excellent electrical conductivity, thermal conductivity, mechanical flexibility and low coefficient of thermal expansion behavior, making it suitable for use in various fields [104]. Similarly, metals can transmit, reflect and absorb EMI and are good electrical conductors. Plastics and rubbers are transparent to EMI and are nonconductive. Metals have the ability to transmit heat and electricity which makes them good for many applications [105]. Various methods have been used to synthesize the shielding composites with the combination of graphene and metals or both with some other materials. A brief description of such methods and the formed composites with shielding effectiveness (SE) has been presented in Table 2. The negative value in the shielding effectiveness (SE) column shows the reflection loss, whereas the positive value is the absorption/total shielding effectiveness.

It can be observed that for the preparation of graphene- and metal-based composites various methods have been utilized. Interestingly, graphene and metals family materials were constructed with different methods, illustrating that the structure of the material significantly depends on the selected method. Hydrothermal and solvothermal are the two most common methods that have been used extensively for these composites. The composites formed by these methods were tested up to the Ku-band frequency range, where the highest reflection loss of $-55.02 \mathrm{~dB}$ was achieved using hydrothermal and reflection loss of $-59.23 \mathrm{~dB}$ was achieved using the solvothermal method. The highest shielding effectiveness of $52.4 \mathrm{~dB}$ in X-band was achieved via using the scalable method. The higher shielding of $60.95 \mathrm{~dB}$ was achieved in THF-band by alternating deposition where graphene and copper were synthesized. In this case, the role of materials properties also gives significant input. Figure 5 shows the maximum shielding effectiveness achieved by utilizing the various methods in different frequency ranges. 
Table 2. Preparation Methods of Graphene-, Metal- and Polymer-Based Composites.

\begin{tabular}{|c|c|c|c|c|c|}
\hline S. No & Method & Material Composite & SE (dB) & Frequency & Reference \\
\hline \multirow{4}{*}{1} & \multirow{4}{*}{ Chemical vapor deposition } & 3DG/Cu & 32.3 & Ku-band & [27] \\
\hline & & 3D Graphene Network@PDMS & 90 & X-band & [25] \\
\hline & & \multirow{2}{*}{ MXene $\left(\mathrm{Ti}_{3} \mathrm{C}_{2} \mathrm{~T}_{\mathrm{X}}\right)$ /graphene/PDMS } & 80 & X-band & \multirow{2}{*}{ [26] } \\
\hline & & & 77 & Ka-band & \\
\hline 2 & Alternating deposition & $\mathrm{Cu} / \mathrm{Gr}$ & 60.95 & THF-band & [28] \\
\hline 3 & Electrophoretic deposition & $\mathrm{Cu}-\mathrm{Ni}-\mathrm{GNS}$ & 42 & X-band & [29] \\
\hline \multirow{13}{*}{4} & \multirow{13}{*}{ In situ growth } & CuNW@G & 52.5 & Ku-band & [39] \\
\hline & & GNP@PANI & -14.5 & X-band & {$[30]$} \\
\hline & & Graphene@NiO@PANI@Ag & -37.5 & Ku-band & [31] \\
\hline & & $\mathrm{TiO}_{2} / \mathrm{PANI} / \mathrm{GO}$ & -51.7 & Ku-band & [34] \\
\hline & & Ag@Graphene/PANI & 29.33 & L-band & [35] \\
\hline & & PANI $/ \mathrm{Li}_{0.5} \mathrm{Fe}_{0.5-\mathrm{x}} \mathrm{Gd}_{\mathrm{x}} \mathrm{O}_{4}$ & 42 & X-band & {$[36]$} \\
\hline & & RGO@Hematite/PVDF & -43.97 & Ku-band & [37] \\
\hline & & $\gamma-\mathrm{Fe}_{2} \mathrm{O}_{3} / \mathrm{RGO} / \mathrm{PANI}$ & 51 & X-band & {$[33]$} \\
\hline & & $\mathrm{PEDOT} / \mathrm{RGO} / \mathrm{SrFe}_{12} \mathrm{O}_{19}$ & 62 & X-band & {$[38]$} \\
\hline & & FeCo@RGO@PPy & -40.7 & Ku-band & [31] \\
\hline & & Graphene/Ni & 20 & X-band & [40] \\
\hline & & $\mathrm{PG}-\mathrm{Fe}_{3} \mathrm{O}_{4}$ & -53 & C-band & {$[41]$} \\
\hline & & G-PANI & 32.5 & Ku-band & {$[32]$} \\
\hline \multirow{2}{*}{5} & \multirow{2}{*}{ Thermal annealing method } & Graphene/Ni hybrid mesh & 26.6 & UHF-band & [43] \\
\hline & & CuNWs-TAGA/Epoxy & 47 & X-band & [42] \\
\hline \multirow{4}{*}{6} & \multirow{4}{*}{ Facile synthetic route } & RGO/Ni hybrid & 52 & Ku-band & [47] \\
\hline & & Polycarbonate/GNP & 47 & X-band & [44] \\
\hline & & $\mathrm{Fe}_{3} \mathrm{O}_{4} / \mathrm{PANI} \mathrm{rod} / \mathrm{RGO}$ & -33.3 & Ku-band & {$[45]$} \\
\hline & & $\mathrm{GNSs}_{-} \mathrm{Fe}_{3} \mathrm{O}_{4} / \mathrm{PVDF}$ & 52 & X-band & {$[46]$} \\
\hline \multirow{10}{*}{7} & \multirow{10}{*}{ Hydrothermal method } & $\mathrm{ZnFe}_{2} \mathrm{O}_{4} @$ graphene@ $\mathrm{TiO}_{2}$ & -55 & S-band & {$[41]$} \\
\hline & & $\mathrm{MoS}_{2}-\mathrm{RGO} / \mathrm{CoFe}_{2} \mathrm{O}_{4}$ & 19.26 & X-band & [53] \\
\hline & & $\mathrm{Fe}_{3} \mathrm{O}_{4} @ \mathrm{C} @$ Graphene & -55.02 & Ku-band & {$[54]$} \\
\hline & & G-F & 20 & Ku-band & [55] \\
\hline & & $\mathrm{Ni}_{0.5} \mathrm{Co}_{0.5} \mathrm{Fe}_{2} \mathrm{O}_{4} /$ graphene & -30.92 & L-band & {$[56]$} \\
\hline & & Graphene@PANI@TiO 2 & -45.4 & Ku-band & {$[48]$} \\
\hline & & GA/PDMS & 60 & Ku-band & [49] \\
\hline & & RGO@CuS@PVDF & -25 & Ku-band & [50] \\
\hline & & G/Polyurethane sponge & 35 & X-band & [51] \\
\hline & & PEDOT:PSS-Fe ${ }_{3} \mathrm{O}_{4}-\mathrm{RGO}$ & -61.4 & Ku-band & {$[52]$} \\
\hline 8 & Scalable method & $\begin{array}{l}\text { Cellulose/reduced graphene oxide } \\
(\mathrm{RGO}) / \mathrm{Fe}_{3} \mathrm{O}_{4} \text { aerogels }\end{array}$ & 52.4 & X-band & [57] \\
\hline
\end{tabular}


Table 2. Cont.

\begin{tabular}{|c|c|c|c|c|c|}
\hline S. No & Method & Material Composite & SE (dB) & Frequency & Reference \\
\hline \multirow{8}{*}{9} & \multirow{8}{*}{ Solvothermal method } & $\mathrm{NiFe}_{2} \mathrm{O}_{4} / \mathrm{RGO}$ & 38.2 & X-band & [60] \\
\hline & & $\mathrm{Fe}_{3} \mathrm{O}_{4} @ f-G N P s$ & 25 & X-band & {$[61]$} \\
\hline & & $\mathrm{Ag} @ \mathrm{Fe}_{3} \mathrm{O}_{4} @ \mathrm{RGO}$ & -40.05 & Ku-band & [62] \\
\hline & & $\mathrm{Fe}_{3} \mathrm{O}_{4} @ \mathrm{C} / \mathrm{RGO}$ & -59.23 & Ku-band & [63] \\
\hline & & Hollow $\mathrm{Fe}_{3} \mathrm{O}_{4} @ G F @ P D M S$ & 70.3 & X-band & [58] \\
\hline & & RGO-PEDOT-NiFe ${ }_{2} \mathrm{O}_{4}$ & -45.4 & \multirow{3}{*}{ Ku-band } & \multirow{3}{*}{ [59] } \\
\hline & & RGO-PANI-NiFe ${ }_{2} \mathrm{O}_{4}$ & -49.7 & & \\
\hline & & RGO-PPy-NiFe ${ }_{2} \mathrm{O}_{4}$ & -44.8 & & \\
\hline 10 & $\begin{array}{l}\text { Filtration-assisted } \\
\text { self-assembly method }\end{array}$ & $\mathrm{Fe}_{3} \mathrm{O}_{4}-\mathrm{C}, \mathrm{C}-\mathrm{MIL}-88 \mathrm{~B} / \mathrm{GNP}$ & 28 & X-band & [64] \\
\hline 11 & Wet stirring process & GO@CIP & -56.4 & Ku-band & [65] \\
\hline \multirow{2}{*}{12} & \multirow{2}{*}{ Self-assembly method } & NRMG & 26.4 & X-band & \\
\hline & & PMMA/RGO & 63.2 & X-band & {$[66]$} \\
\hline 13 & $\begin{array}{l}\text { Vacuum-assisted } \\
\text { filtration method }\end{array}$ & $\mathrm{RGO} / \mathrm{CNF} @ \mathrm{Ag}-\mathrm{Fe}_{3} \mathrm{O}_{4}$ & 21 & X-band & [67] \\
\hline \multirow{9}{*}{14} & \multirow{9}{*}{ Solution processing method } & PVC/PANI/GNP & 51 & K-band & [74] \\
\hline & & Gn/SiCnw/PVDF & 32.5 & X-band & [68] \\
\hline & & PVDF/graphene & 47 & X-band & [69] \\
\hline & & $\mathrm{Fe}_{3} \mathrm{O}_{4} @ \mathrm{RGO} / \mathrm{TPU}$ & $\sim 15.51 \pm 1.6$ & X-band & [70] \\
\hline & & BaFe@TRGO@TPU & -61 & K-band & [71] \\
\hline & & $\mathrm{Fe}_{3} \mathrm{O}_{4} @ S L G A P C @ P V A$ & 20 & X-band & [72] \\
\hline & & PVDF/GNP-Ni-CNT & 46.4 & Ku-band & [73] \\
\hline & & $\mathrm{PVDF} / \mathrm{PFC}$ & -29.7 & Ku-band & [75] \\
\hline & & TPU/TRG & 32 & Ku-band & [76] \\
\hline \multirow{4}{*}{15} & \multirow{4}{*}{$\begin{array}{l}\text { Chemical oxidative } \\
\text { polymerization }\end{array}$} & Graphene@ $\mathrm{Fe}_{3} \mathrm{O}_{4} @ \mathrm{PANI} @ \mathrm{WO}_{3}$ & -46.7 & X-band & {$[81]$} \\
\hline & & $\mathrm{PEDOT} / \mathrm{RGO} / \mathrm{PbTiO}_{3}$ & 51.94 & Ku-band & [79] \\
\hline & & $\mathrm{Fe}_{3} \mathrm{O}_{4} / \mathrm{C}: \mathrm{PPy}$ & $>28$ & C-band & {$[80]$} \\
\hline & & Polypyrrole/BST/RGO/ $\mathrm{Fe}_{3} \mathrm{O}_{4}$ & 48 & X-band & [78] \\
\hline \multirow{2}{*}{16} & \multirow{2}{*}{ Co-precipitation method } & $\mathrm{Ti}_{3} \mathrm{C}_{2} \mathrm{~T}_{\mathrm{x}} / \mathrm{Fe}_{3} \mathrm{O}_{4} @ \mathrm{PANI}$ & 58.8 & X-band & {$[82]$} \\
\hline & & $\mathrm{GNP} / \mathrm{Fe}_{3} \mathrm{O}_{4} /$ Epoxy & 37.03 & X-band & [83] \\
\hline 17 & Centrifugal mixing method & TGO/CI/Epoxy & 40 & X-band & [84] \\
\hline 18 & Citrate precursor method & PANI/BF/RGO & 31.1 & X-band & [85] \\
\hline 19 & Chemical reduction & RGO-CF/EP & 37.6 & X-band & [15] \\
\hline 20 & Hot-molding process & $\mathrm{PVDF} / \mathrm{n}-\mathrm{Fe}$ & 40.21 & Ku-band & [86] \\
\hline 21 & Mechanical mixing & Graphene flakes@PDMS & 31 & THF-band & [87] \\
\hline 22 & Dilute polymerization & Graphene@ $\mathrm{Fe}_{3} \mathrm{O}_{4} @ \mathrm{SiO}_{2} @$ polyaniline & -40.7 & X-band & {$[88]$} \\
\hline 23 & $\begin{array}{l}\text { High-pressure solid-phase } \\
\text { compression molding }\end{array}$ & RGO@polystyrene & 45.1 & X-band & [88] \\
\hline 24 & Injection molding process & Polyethylene@GNP & 31 & K-band & [89] \\
\hline \multirow{3}{*}{25} & \multirow{3}{*}{ Ultrasonication technique } & GNP/EPDM & 35 & Ku-band & [90] \\
\hline & & Ni@GNS@PVDF & 51.4 & K-band & [91] \\
\hline & & GNP/Fe/Epoxy & -78 & V-band & [92] \\
\hline
\end{tabular}


Table 2. Cont.

\begin{tabular}{|c|c|c|c|c|c|}
\hline S. No & Method & Material Composite & SE (dB) & Frequency & Reference \\
\hline \multirow{5}{*}{26} & \multirow{5}{*}{ Hummer's method } & $\mathrm{PANI} / \mathrm{GO} / \mathrm{Fe}_{3} \mathrm{O}_{4}$ & -53.5 & Ku-band & [93] \\
\hline & & Cu@RGOFM@PDMS & 74.2 & X-band & [94] \\
\hline & & $\mathrm{GCF} / \mathrm{MG}_{3} / \mathrm{EP}$ & 51.1 & K-band & [95] \\
\hline & & RGO@PEI & 26 & X-band & [96] \\
\hline & & $\mathrm{PVA} / \mathrm{Gr} / \mathrm{Fe}_{3} \mathrm{O}_{4}$ & 40.7 & X-band & [97] \\
\hline \multirow{7}{*}{27} & \multirow{7}{*}{ Hot compressed method } & PMMA/graphene & 21 & X-band & \multirow{2}{*}{ [99] } \\
\hline & & PVC/graphene & 31 & X-band & \\
\hline & & Polylactic acid/Biochar/Graphite & 30 & K-band & [14] \\
\hline & & $\mathrm{NiFe}_{2} \mathrm{O}_{4}$-RGO-Polypropylene & 29.4 & C-band & [100] \\
\hline & & GNP/CLF/PEEK & 27.1 & X-band & [101] \\
\hline & & Conventional RGO/PS & 14.2 & K-band & \multirow{2}{*}{ [98] } \\
\hline & & Segregated RGO/PS & 29.7 & K-band & \\
\hline 28 & 3D printing method & Graphene $/ \mathrm{Li}_{0.35} \mathrm{Zn}_{0.3} \mathrm{Fe}_{0.35} \mathrm{O}_{4} / \mathrm{PMMA}$ & -46.1 & Ku-band & [102] \\
\hline
\end{tabular}

Note: Ultra high frequency $(\mathrm{UHF})=300 \mathrm{MHz}-1 \mathrm{GHz}, \mathrm{L}-$ band $=1$ to $2 \mathrm{GHz}$, S-band $=2 \mathrm{GHz}-4 \mathrm{GHz}, \mathrm{C}-\mathrm{band}=4 \mathrm{GHz}-8 \mathrm{GHz}$, X-band $=8 \mathrm{GHz}-12 \mathrm{GHz}, \mathrm{Ku}$-band $=12 \mathrm{GHz}-18 \mathrm{GHz}, \mathrm{K}$-band $=18 \mathrm{GHz}-27 \mathrm{GHz}, \mathrm{Ka}$-band $=27 \mathrm{GHz}-40 \mathrm{GHz}, \mathrm{V}-$ band $=40 \mathrm{GHz}-75 \mathrm{GHz}$, Tremendously High Frequency (THF) $=300-3000 \mathrm{GHz}$.

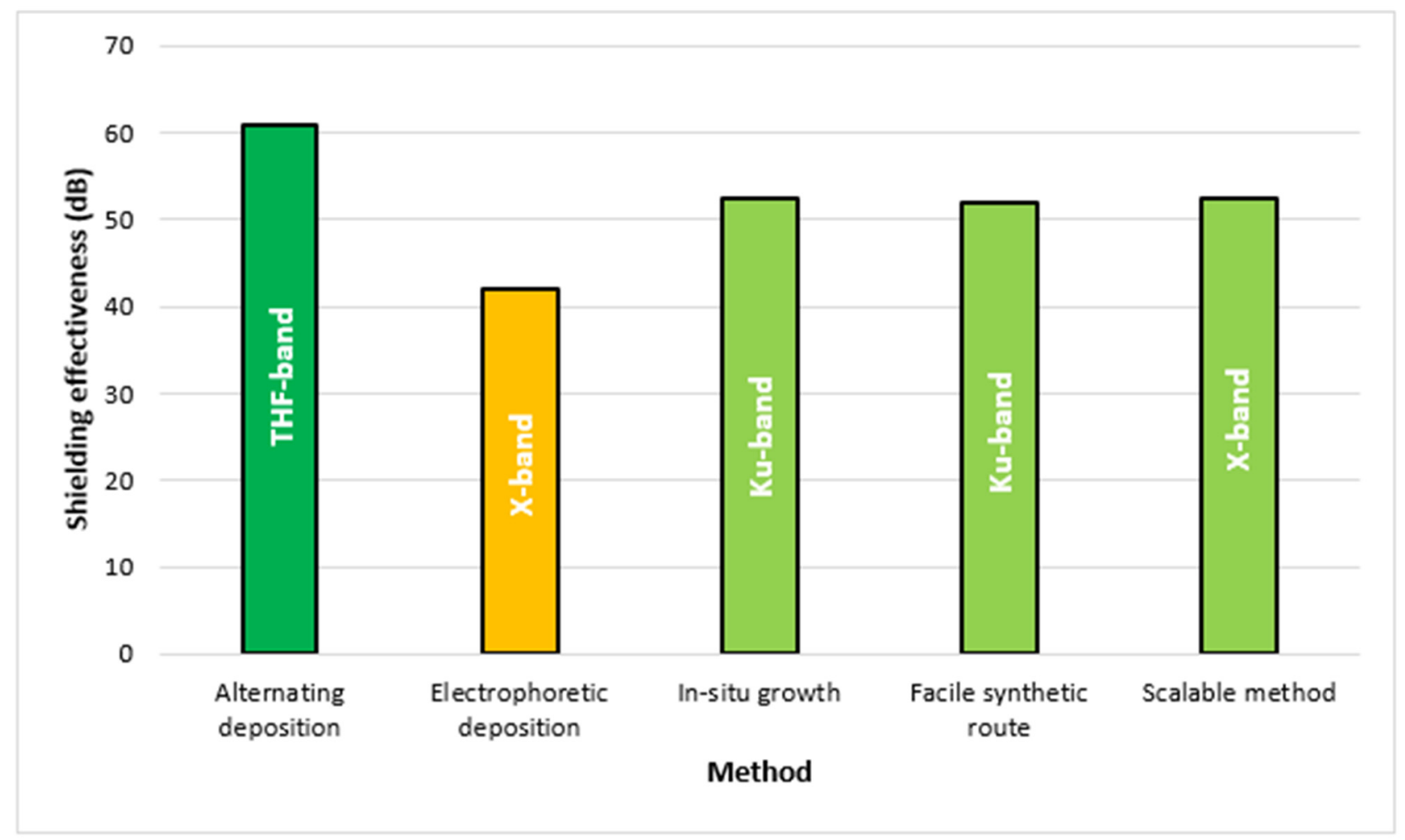

Figure 5. Top 5 methods providing higher SE in graphene- and metal-based composites.

It can be observed that in situ growth, facile synthetic route and scalable method give SE greater than $50 \mathrm{~dB}$ in X-band and Ku-band frequency range, whereas, with electrophoretic deposition, the SE was in the range of $40 \mathrm{~dB}$ in $\mathrm{X}$-band. Looking into the combinations of the materials, a scalable method provides better shielding in X-band, while both in situ growth and facile synthetic route come as the most suitable methods for Ku-band frequency range materials. The highest shielding effectiveness, greater than 
$60 \mathrm{~dB}$, was achieved by alternating deposition in THF-band; however, this method requires further exploration.

\subsubsection{Methods for Preparation of Polymer-Based Composites}

Graphene and metals although are the most suitable composites for EMI shielding but have some limitations $[106,107]$. Due to the advancement in electronic applications, the demand for an effective shielding material has also boost up where thermal expansion, material design flexibility, and non-corrosive properties play a significant role. Besides these properties, the weight-to-strength ratio of EMI shielding materials is also important from the inertia and structural perspective. Moreover, to be part of the electronic system, the material should be lightweight where the polymer composite materials emerge as the most promising materials [108]. Forming a polymer composite, various methods have been used as shown in Table 2.

As shown in Table 2, to form a polymer-based composite, various methods have been used where the most adopted methods are solution processing method, in situ growth, hydrothermal method, Hummer's method and solvothermal method. In the $\mathrm{X}$-band frequency range, the highest total shielding effectiveness was achieved up to $90 \mathrm{~dB}$ by utilizing chemical vapor deposition. In Ku-band, the highest total shielding effectiveness was achieved up to $60 \mathrm{~dB}$ with a reflection loss of $-61.4 \mathrm{~dB}$ by making a composite with the hydrothermal method. In the K-band frequency range the maximum total shielding effectiveness of $51.4 \mathrm{~dB}, 51.1 \mathrm{~dB}$ and $51 \mathrm{~dB}$ by using ultrasonication technique, Hummer's method and solution processing method. While a reflection loss of $-61 \mathrm{~dB}$ was achieved via the solution processing method. In the Ka-band frequency range, the highest total shielding effectiveness of $77 \mathrm{~dB}$ was achieved by using chemical vapor deposition. Overall, this method gave better shielding in both X-band and Ka-band. A high reflection loss of $-78 \mathrm{~dB}$ was also observed in the $\mathrm{V}$-band frequency range by the ultrasonication method. A comparison of all the methods has been drawn in Figure 6 which gives shielding effectiveness greater than $50 \mathrm{~dB}$ in their respectable frequency ranges.

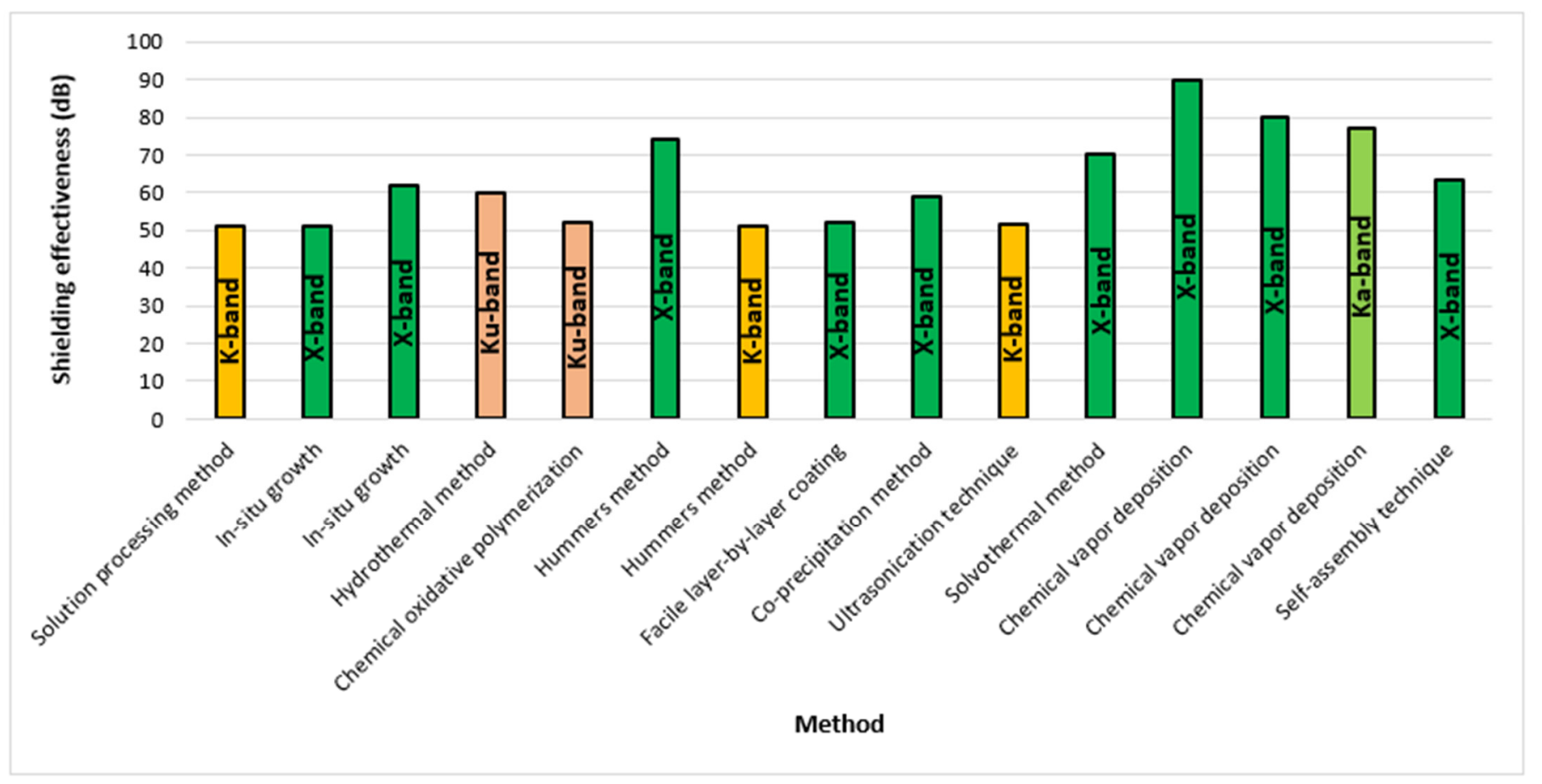

Figure 6. Top methods providing higher SE in polymer-based composites.

In the X-band frequency range the highest shielding effectiveness was attained by forming the polymer composite via chemical vapor deposition. In the Ku-band frequency 
range, the hydrothermal method was more efficient as compared to chemical oxidative polymerization. In the K-band frequency range, solution processing method, Hummer's method and ultrasonication technique, all performed efficiently, while Ka-band chemical vapor deposition gave better shielding effectiveness. The overall maximum shielding was achieved by chemical vapor deposition; however, more combinations need to be tested formed by this method.

Although this review is limited to the existing methods for composite synthesis, it is worth mentioning that reviewing materials properties is of importance as discussed in [8] and [19]. For instance, the concentration of graphene, density of porous materials, amount of filler content and the thickness of the composite impacts an improvement in the shielding effectiveness. Before proceeding towards the method adaptation, it is important to have an in-dept understanding of the properties of the materials as well, as the authors discussed in [24]. Various studies showed that by increasing the graphene loading and composite thickness, at some extent the shielding effectiveness increases. Achieving a desirable output, the role of morphology cannot be neglected. In this review, the authors tried to draw a comparison of the composite's formation methods for better understanding for future researchers, while performing the review it was observed that this area still needs exploration in terms of comparison of the same composite formed via different methods. In this way, the direction for each composite will be clearer for researchers.

\subsection{Artificial Intelligence Methods}

Development of artificial intelligence, mainly in machine learning, a variety of reforms have been made in materials formation by exploring new materials and their combinations, along with their properties. This approach is a trending topic as a lot of work is still ongoing [109-111]. The machine learning method has the potential to discover the properties of new composites [112]. However, its benefits are still unrevealed, especially in polymer science $[113,114]$. Various properties of polymers depend on the degree of crystallinity. Machine learning-based methods are competent enough to forecast crystallinity, which can counter the deficiency of traditional methods. With the help of machine learning, melting temperature can also be predicted for new polymers, as its one of the difficult parameters to be controlled in traditional methods [115]. For specific applications, machine learning models are used to identify the properties of the polymer, such as dielectric constant [116] which is a parameter for attaining efficient EMI shielding.

The composite synthesis requires materials recognition to attain the desirable properties required for specific applications. The traditional methods have been used extensively to evaluate the required properties, based on which further assessment is performed [117]. However, there are various drawbacks of these traditional methods such as time and material consumption and selection of appropriate method for a specific material $[118,119]$. In this manner, machine learning is a tool that can be utilized for quick decisions [120]. It not only helps in finding the properties, but new material formation is also possible by its adaptation [113,121]. Various machine learning approaches that have been used in EMI are presented in Table 3. 
Table 3. Machine learning methods for EMI.

\begin{tabular}{|c|c|c|c|c|c|}
\hline S. No & $\begin{array}{l}\text { Machine Learning } \\
\text { Method }\end{array}$ & Reference & Remarks & Advantage & Disadvantage \\
\hline 1 & $\begin{array}{l}\text { Association rule } \\
\text { learning and } \\
\text { decision tree } \\
\text { algorithm }\end{array}$ & [122] & $\begin{array}{l}\text { Effective in dealing with } \\
\text { electromagnetic interference } \\
\text { in high power line } \\
\text { communication and helps to } \\
\text { eliminate the troubleshooting }\end{array}$ & $\begin{array}{l}\text { - Normalization and } \\
\text { data scaling not } \\
\text { required }\end{array}$ & - $\quad$ Time consuming \\
\hline 2 & $\begin{array}{l}\text { K-nearest neighbors } \\
\text { (k-NN) algorithm }\end{array}$ & [123] & $\begin{array}{l}\text { Continuous monitoring in air } \\
\text { traffic control communication } \\
\text { is applicable against } \\
\text { electromagnetic interference }\end{array}$ & $\begin{array}{ll}\text { - } & \text { Training period not } \\
& \text { required } \\
\text { - } & \text { Easy } \\
& \text { implementation }\end{array}$ & $\begin{array}{l}\text { - } \\
\text { large and huitable for } \\
\text { dimension data }\end{array}$ \\
\hline 3 & $\begin{array}{l}\text { Artificial Neural } \\
\text { Networks }\end{array}$ & [124-128] & $\begin{array}{l}\text { An effective approach to } \\
\text { eliminate electromagnetic } \\
\text { interference problems }\end{array}$ & - $\quad$ Fast evaluation & $\begin{array}{l}\text { Problem } \\
\text { identification is } \\
\text { difficult }\end{array}$ \\
\hline 4 & $\begin{array}{l}\text { Back-propagation } \\
\text { Neural Network }\end{array}$ & {$[129,130]$} & $\begin{array}{l}\text { Use backward pass approach } \\
\text { for parameters adjustment }\end{array}$ & $\begin{array}{l}\text { - } \quad \text { Fast and easy } \\
\text { to use }\end{array}$ & $\begin{array}{l}\text { - Actual } \\
\text { performance } \\
\text { depends on data } \\
\text { input for } \\
\text { problem solving }\end{array}$ \\
\hline 5 & $\begin{array}{c}\text { Self-Organizing } \\
\text { Feature Map Neural } \\
\text { Network }\end{array}$ & {$[131,132]$} & $\begin{array}{l}\text { Effective in evaluating the } \\
\text { global features of } \\
\text { electromagnetic inferences } \\
\text { factors }\end{array}$ & $\begin{array}{l}\text { Interpretation of } \\
\text { data is easy } \\
\text { Grid clustering is } \\
\text { helpful to evaluate } \\
\text { the data similarity }\end{array}$ & - $\quad$ Slow training \\
\hline 6 & Neural Networks & [133-137] & $\begin{array}{l}\text { Effective for electromagnetic } \\
\text { interference generated } \\
\text { underground metallic } \\
\text { pipelines, high voltage power } \\
\text { lines and other problems }\end{array}$ & $\begin{array}{ll} & \text { Detect complex } \\
& \text { nonlinear } \\
& \text { relationship } \\
\text { - } & \text { Suitable for } \\
& \text { multiple training }\end{array}$ & - $\quad$ Black box \\
\hline 7 & Monte Carlo Method & {$[138,139]$} & $\begin{array}{l}\text { Effective in electromagnetic } \\
\text { interference problems solving } \\
\text { especially in power lines }\end{array}$ & - $\quad$ Flexible simulation & - $\quad$ Time consuming \\
\hline
\end{tabular}

It is evident from the above table that various machine learning approaches are available to deal with EMI problems; however, this area requires extensive work as most researchers are focusing on the traditional methods, regardless of their time and cost consuming drawbacks instead of adopting the artificial intelligence. Machine learning methods to evaluate the composite properties and formation need vital attention are the future in material science to bring major reforms by constructing new composites. With the help of machine learning, material properties can be pre-tested, which can be helpful in the construction of the best-suited combination before its experimental formation. Another approach that can be utilized with the help of machine learning is the formation of 3D shielding materials constructed via 3D printing. Such $3 \mathrm{D}$ printing can be a time and material saver. The results further reveal that within the past half a decade the machine learning methods including artificial neural networks had brought significant improvement for modelling EMI materials. There is a research trend in the direction of using advanced forms of machine learning for comparative analysis, research and development employing hybrid and ensemble machine learning methods to deliver higher performance. ANN and advanced forms of neural networks and optimized ANN are the most dominant machine learning methods used as discussed in [140,141]. 


\section{Future Direction}

The dependency of the traditional method is mainly on the structure of the material which has been taken to form a composite material. Moreover, many new methods have been introduced which need further investigation. Although there are many methods available for the formation of shielding material, very few are effective in giving desirable results. However, the area is still unexplored in terms of methods comparison. The opinion of method selection can be biased as most researchers present their findings as extraordinary without comparison. The most suitable way to observe the efficiency of a particular method for any composite is to make the same composite with different available methods and then perform the same analysis, which will give the true picture of the adopted methods. Moreover, the inclusion of machine learning in EMI applications can bring reform. This is still a gap in this area of knowledge and the investigation will be a benchmark for new researchers as it is time-consuming and costly if a wrong method is adopted. The results further reveal that within the past half-decade machine learning methods, including artificial neural networks, have brought significant improvement for modelling EMI materials. There is a research trend in using advanced forms of machine learning for comparative analysis, research and development employing hybrid and ensemble machine learning methods to deliver higher performance.

\section{Conclusions}

This study is a review of the formation of graphene-, metal-, and polymer-based composites via various traditional and artificial intelligence methods. The working on graphene- and metal-based composites as shielding material has existd for a long time, while the addition of polymer-based composites is new and remarkable results have been seen in the field of electromagnetic shielding. An extensive literature review was conducted where it was revealed that in graphene- and metal-based composites, the alternating deposition method, which is still less explored, provides the maximum shielding effectiveness in THF-band. In Ku-band, in situ growth, while in X-band, scalable method utilization, provide better shielding effectiveness. In polymer-based composites, the highest shielding effectiveness came when the composite was formed from chemical vapor deposition in X-band. In Ku-band hydrothermal method, in K-band solution processing method, Hummer's method and ultrasonication technique and in Ka-band chemical vapor deposition utilization provided better shielding effectiveness. However, there is still a gap in the implication of machine learning in EMI applications. The review was conducted with the purpose to highlight the best-suited method for the formation of the composites; however, it is concluded that it is too early to declare any method as the best as there is still a gap in this area of knowledge that needs to be filled by making extensive research in which a comparison of the methods should be made for a single composite. The formation of a single composite via various methods upon shielding effectiveness will reveal which is the most suitable method among the available list in providing the highest shielding effectiveness. The results further reveal that within the past half-decade machine learning methods including artificial neural networks have brought significant improvement for modelling EMI materials. There is a research trend of using advanced forms of machine learning for comparative analysis, research and development employing hybrid and ensemble machine learning methods to deliver higher performance.

Author Contributions: Conceptualization, S.A., B.H.G. and F.A.; methodology, S.A., B.H.G. and F.A.; software, S.A.; validation, B.H.G., F.A., M.F.J. and I.F.; formal analysis, S.A., B.H.G. and F.A.; investigation, S.A.; resources, B.H.G., F.A. and A.M.; data curation, S.A.; writing-original draft preparation, S.A.; writing - review and editing, B.H.G., F.A., A.M. and I.F.; visualization, A.M.; supervision, B.H.G., F.A., I.F. and A.M.; project administration, B.H.G., F.A. and A.M.; funding acquisition, B.H.G. and A.M. All authors have read and agreed to the published version of the manuscript.

Funding: This research has not received any external funding.

Data Availability Statement: Data is available upon request. 
Acknowledgments: The authors would like to thank Universiti Teknologi PETRONAS (UTP) for the support provided for this research. Mosavi and Felde would like to acknowledge the support from the project GINOP-2.2.1-18-2018-00015. Felde would like to acknowledge his support by the 2019-2.1.11-TÉT-2019-00007 Hungarian-Mexican bilateral project.

Conflicts of Interest: The authors declare no conflict of interest.

\section{References}

1. Raagulan, K.; Kim, B.M.; Chai, K.Y. Recent Advancement of Electromagnetic Interference (EMI) Shielding of Two Dimensional (2D) MXene and Graphene Aerogel Composites. Nanomaterials 2020, 10, 702. [CrossRef]

2. Li, Y.; Chen, S.; Zhao, K.; Gui, Y.; Fang, S.; Xu, Y.; Ma, Z. Effects of electromagnetic radiation on health and immune function of operators. Chin. J. Ind. Hyg. Occup. Dis. 2013, 31, 602-605.

3. Chung, D. Electromagnetic interference shielding effectiveness of carbon materials. Carbon 2001, 39, 279-285. [CrossRef]

4. Cheng, K.; Ramakrishna, S.; Lee, K. Electromagnetic shielding effectiveness of copper/glass fiber knitted fabric reinforced polypropylene composites. Compos. Part A Appl. Sci. Manuf. 2000, 31, 1039-1045. [CrossRef]

5. Joo, J.; Epstein, A. Electromagnetic radiation shielding by intrinsically conducting polymers. Appl. Phys. Lett. 1994, 65, 2278-2280. [CrossRef]

6. Saini, P. Electrical properties and electromagnetic interference shielding response of electrically conducting thermosetting nanocomposites. Nanocomposites 2013. [CrossRef]

7. Saini, P. Historical review of advanced materials for electromagnetic interference (EMI) shielding: Conjugated polymers, carbon nanotubes, graphene based composites. Indian J. Pure Appl. Phys. (IJPAP) 2019, 57, 338-351.

8. Bellucci, S.; Bistarelli, S.; Cataldo, A.; Micciulla, F.; Kranauskaite, I.; Macutkevic, J.; Banys, J.; Volynets, N.; Paddubskaya, A.; Bychanok, D. Broadband dielectric spectroscopy of composites filled with various carbon materials. IEEE Trans. Microw. Theory Tech. 2015, 63, 2024-2031. [CrossRef]

9. Wiley. Advance Materials for Electromagnetic Sheilding. 2019. Available online: https://books.google.com.my/books? id=kGVDwAAQBAJ\&lpg=PA1\&ots=RhoUSihwqR\&dq=EMI\%20related\%20materials\%20and\%20graphs\&lr\&pg=PP21\#v= onepage\&q=EMI\%20related $\% 20$ materials $\% 20$ and $\% 20$ graphs \&f=false (accessed on 8 April 2021).

10. Yang, S.; Lozano, K.; Lomeli, A.; Foltz, H.D.; Jones, R. Electromagnetic interference shielding effectiveness of carbon nanofiber/LCP composites. Compos. Part A Appl. Sci. Manuf. 2005, 36, 691-697. [CrossRef]

11. Von Klemperer, C.J.; Maharaj, D. Composite electromagnetic interference shielding materials for aerospace applications. Compos. Struct. 2009, 91, 467-472. [CrossRef]

12. Wanasinghe, D.; Aslani, F. A review on recent advancement of electromagnetic interference shielding novel metallic materials and processes. Compos. Part B Eng. 2019, 176, 107207. [CrossRef]

13. Geetha, S.; Satheesh Kumar, K.; Rao, C.R.; Vijayan, M.; Trivedi, D. EMI shielding: Methods and materials-A review. J. Appl. Polym. Sci. 2009, 112, 2073-2086. [CrossRef]

14. Tolvanen, J.; Hannu, J.; Hietala, M.; Kordas, K.; Jantunen, H. Biodegradable multiphase poly (lactic acid)/biochar/graphite composites for electromagnetic interference shielding. Compos. Sci. Technol. 2019, 181, 107704. [CrossRef]

15. Wu, J.; Chen, J.; Zhao, Y.; Liu, W.; Zhang, W. Effect of electrophoretic condition on the electromagnetic interference shielding performance of reduced graphene oxide-carbon fiber/epoxy resin composites. Compos. Part B Eng. 2016, 105, 167-175. [CrossRef]

16. Cheaptubes.com. Graphene Synthesis, Properties, and Applications. 2020. Available online: https://www.cheaptubes.com/ graphene-synthesis-properties-and-applications/ (accessed on 8 April 2021).

17. Ayub, S.; Guan, B.H.; Ahmad, F. Graphene and Iron based composites as EMI shielding: A Systematic Review. In Proceedings of the 2020 Second International Sustainability and Resilience Conference: Technology and Innovation in Building Designs (51154), Sakheer, Bahrain, 11-12 November 2020; pp. 1-5.

18. Tjaronge, M.W.; Musarat, M.A.; Law, K.; Alaloul, W.S.; Ayub, S. Effect of Graphene Oxide on Mechanical Properties of Rubberized Concrete: A Review. In Proceedings of the International Conference on Civil, Offshore and Environmental Engineering; Springer: Singapore, 2021.

19. Plyushch, A.; Macutkevic, J.; Kuzhir, P.; Banys, J.; Bychanok, D.; Lambin, P.; Bistarelli, S.; Cataldo, A.; Micciulla, F.; Bellucci, S. Electromagnetic properties of graphene nanoplatelets/epoxy composites. Compos. Sci. Technol. 2016, 128, 75-83. [CrossRef]

20. Santhosi, B.; Ramji, K.; Rao, N.M. Design and development of polymeric nanocomposite reinforced with graphene for effective EMI shielding in X-band. Phys. B Condens. Matter 2020, 586, 412144. [CrossRef]

21. Kim, B.-J.; Bae, K.-M.; Lee, Y.S.; An, K.-H.; Park, S.-J. EMI shielding behaviors of Ni-coated MWCNTs-filled epoxy matrix nanocomposites. Surf. Coat. Technol. 2014, 242, 125-131. [CrossRef]

22. Ma, Z. Friction stir processing technology: A review. Metall. Mater. Trans. A 2008, 39, 642-658. [CrossRef]

23. Chen, L.-Y.; Konishi, H.; Fehrenbacher, A.; Ma, C.; Xu, J.-Q.; Choi, H.; Xu, H.-F.; Pfefferkorn, F.E.; Li, X.-C. Novel nanoprocessing route for bulk graphene nanoplatelets reinforced metal matrix nanocomposites. Scr. Mater. 2012, 67, 29-32. [CrossRef]

24. Ayub, S.; Guan, B.H.; Ahmad, F.; Oluwatobi, Y.A.; Nisa, Z.U.; Javed, M.F.; Mosavi, A. Graphene and Iron Reinforced Polymer Composite Electromagnetic Shielding Applications: A Review. Polymers 2021. [CrossRef]

25. Ao, D.; Tang, Y.; Xu, X.; Xiang, X.; Yu, J.; Li, S.; Zu, X. Highly Conductive PDMS Composite Mechanically Enhanced with 3D-Graphene Network for High-Performance EMI Shielding Application. Nanomaterials 2020, 10, 768. [CrossRef] 
26. Nguyen, V.-T.; Min, B.K.; Yi, Y.; Kim, S.J.; Choi, C.-G. MXene (Ti3C2TX)/graphene/PDMS composites for multifunctional broadband electromagnetic interference shielding skins. Chem. Eng. J. 2020, 393, 124608. [CrossRef]

27. Cheng, K.; Xiong, W.; Li, Y.; Hao, L.; Yan, C.; Li, Z.; Liu, Z.; Wang, Y.; Essa, K.; Lee, L. In-situ deposition of three-dimensional graphene on selective laser melted copper scaffolds for high performance applications. Compos. Part A Appl. Sci. Manuf. 2020, 135, 105904. [CrossRef]

28. Hou, S.; Ma, W.; Li, G.; Zhang, Y.; Ji, Y.; Fan, F.; Huang, Y. Excellent Terahertz shielding performance of ultrathin flexible $\mathrm{Cu}$ /graphene nanolayered composites with high stability. J. Mater. Sci. Technol. 2020, 52, 136-144. [CrossRef]

29. Ji, K.; Zhao, H.; Huang, Z.; Dai, Z. Performance of open-cell foam of $\mathrm{Cu}-\mathrm{Ni}$ alloy integrated with graphene as a shield against electromagnetic interference. Mater. Lett. 2014, 122, 244-247. [CrossRef]

30. Khasim, S. Polyaniline-Graphene nanoplatelet composite films with improved conductivity for high performance X-band microwave shielding applications. Results Phys. 2019, 12, 1073-1081. [CrossRef]

31. Wang, Y.; Wu, X.; Zhang, W.; Luo, C.; Li, J. Synthesis of ferromagnetic sandwich FeCo@ graphene@ PPy and enhanced electromagnetic wave absorption properties. J. Magn. Magn. Mater. 2017, 443, 358-365. [CrossRef]

32. Zhou, Y.; Zhang, W.; Pan, Z.; Zhao, B. Graphene-doped polyaniline nanocomposites as electromagnetic wave absorbing materials. J. Mater. Sci. Mater. Electron. 2017, 28, 10921-10928. [CrossRef]

33. Singh, A.P.; Mishra, M.; Sambyal, P.; Gupta, B.K.; Singh, B.P.; Chandra, A.; Dhawan, S. Encapsulation of $\gamma-\mathrm{Fe}_{2} \mathrm{O}_{3}$ decorated reduced graphene oxide in polyaniline core-shell tubes as an exceptional tracker for electromagnetic environmental pollution. $J$. Mater. Chem. A 2014, 2, 3581-3593. [CrossRef]

34. Jia, Q.; Wang, W.; Zhao, J.; Xiao, J.; Lu, L.; Fan, H. Synthesis and characterization of $\mathrm{TiO}_{2} /$ polyaniline/graphene oxide bouquet-like composites for enhanced microwave absorption performance. J. Alloys Compd. 2017, 710, 717-724. [CrossRef]

35. Chen, Y.; Li, Y.; Yip, M.; Tai, N. Electromagnetic interference shielding efficiency of polyaniline composites filled with graphene decorated with metallic nanoparticles. Compos. Sci. Technol. 2013, 80, 80-86. [CrossRef]

36. Dar, M.A.; Majid, K.; Farukh, M.; Dhawan, S.; Kotnala, R.; Shah, J. Electromagnetic attributes a dominant factor for the enhanced EMI shielding of PANI/Li0. 5Fe $2.5-\mathrm{xGdxO}_{4}$ core shell structured nanomaterial. Arab. J. Chem. 2019, 12, 5111-5119. [CrossRef]

37. Chen, D.; Quan, H.; Huang, Z.; Luo, S.; Luo, X.; Deng, F.; Jiang, H.; Zeng, G. Electromagnetic and microwave absorbing properties of RGO@ hematite core-shell nanostructure/PVDF composites. Compos. Sci. Technol. 2014, 102, 126-131.

38. Dalal, J.; Lather, S.; Gupta, A.; Tripathi, R.; Maan, A.S.; Singh, K.; Ohlan, A. Reduced Graphene Oxide Functionalized Strontium Ferrite in Poly (3, 4-ethylenedioxythiophene) Conducting Network: A High-Performance EMI Shielding Material. Adv. Mater. Technol. 2019, 4, 1900023. [CrossRef]

39. Wu, S.; Zou, M.; Li, Z.; Chen, D.; Zhang, H.; Yuan, Y.; Pei, Y.; Cao, A. Robust and stable Cu Nanowire@ Graphene core-shell aerogels for ultraeffective electromagnetic interference shielding. Small 2018, 14, 1800634. [CrossRef] [PubMed]

40. Zhang, S.; Wang, Y.; Ran, Q.; Fu, Q.; Gu, Y. Electromagnetic interference shielding property of polybenzoxazine/graphene/nickel composites. React. Funct. Polym. 2019, 143, 104324. [CrossRef]

41. Zheng, Y.; Wang, X.; Wei, S.; Zhang, B.; Yu, M.; Zhao, W.; Liu, J. Fabrication of porous graphene-Fe $\mathrm{O}_{4} \mathrm{O}_{4}$ hrid composites with outstanding microwave absorption performance. Compos. Part A Appl. Sci. Manuf. 2017, 95, 237-247. [CrossRef]

42. Yang, X.; Fan, S.; Li, Y.; Guo, Y.; Li, Y.; Ruan, K.; Zhang, S.; Zhang, J.; Kong, J.; Gu, J. Synchronously improved electromagnetic interference shielding and thermal conductivity for epoxy nanocomposites by constructing 3D copper nanowires/thermally annealed graphene aerogel framework. Compos. Part A Appl. Sci. Manuf. 2020, 128, 105670. [CrossRef]

43. Tran, V.V.; Nguyen, D.D.; Nguyen, A.T.; Hofmann, M.; Hsieh, Y.-P.; Kan, H.-C.; Hsu, C.-C. Electromagnetic Interference Shielding by Transparent Graphene/Nickel Mesh Films. ACS Appl. Nano Mater. 2020, 3, 7474-7481. [CrossRef]

44. Nimbalkar, P.; Korde, A.; Goyal, R. Electromagnetic interference shielding of polycarbonate/GNP nanocomposites in X-band. Mater. Chem. Phys. 2018, 206, 251-258. [CrossRef]

45. Ma, Y.; Zhou, Y.; Xiong, Z.; Sun, Y.; Qi, C.; Zhang, Y.; Liu, Y. Facile synthesis of $\mathrm{Fe}_{3} \mathrm{O}_{4} /$ PANI rod/rGO nanocomposites with giant microwave absorption bandwidth. J. Mater. Sci. Mater. Electron. 2019, 30, 4819-4830. [CrossRef]

46. Guo, Z.; Ren, P.; Fu, B.; Ren, F.; Jin, Y.; Sun, Z. Multi-layered graphene-Fe3O4/poly (vinylidene fluoride) hybrid composite films for high-efficient electromagnetic shielding. Polym. Test. 2020, 89, 106652. [CrossRef]

47. Xu, W.; Wang, G.-S.; Yin, P.-G. Designed fabrication of reduced graphene oxides/Ni hybrids for effective electromagnetic absorption and shielding. Carbon 2018, 139, 759-767. [CrossRef]

48. Liu, P.; Huang, Y.; Yan, J.; Zhao, Y. Magnetic graphene@ PANI@ porous $\mathrm{TiO}_{2}$ ternary composites for high-performance electromagnetic wave absorption. J. Mater. Chem. C 2016, 4, 6362-6370. [CrossRef]

49. Ni, J.; Zhan, R.; Qiu, J.; Fan, J.; Dong, B.; Guo, Z. Multi-interfaced graphene aerogel/polydimethylsiloxane metacomposites with tunable electrical conductivity for enhanced electromagnetic interference shielding. J. Mater. Chem. C 2020, 8, 11748-11759. [CrossRef]

50. Sharma, D.; Menon, A.V.; Bose, S. Graphene templated growth of copper sulphide 'flowers' can suppress electromagnetic interference. Nanoscale Adv. 2020, 2, 3292-3303. [CrossRef]

51. Hu, Z.; Ji, X.; Li, B.; Luo, Y. A self-assembled graphene/polyurethane sponge for excellent electromagnetic interference shielding performance. RSC Adv. 2019, 9, 25829-25835. [CrossRef] 
52. Wang, X.; Shu, J.-C.; He, X.-M.; Zhang, M.; Wang, X.-X.; Gao, C.; Yuan, J.; Cao, M.-S. Green approach to conductive PEDOT: PSS decorating magnetic-graphene to recover conductivity for highly efficient absorption. ACS Sustain. Chem. Eng. 2018, 6, 14017-14025. [CrossRef]

53. Prasad, J.; Singh, A.K.; Haldar, K.K.; Tomar, M.; Gupta, V.; Singh, K. $\mathrm{CoFe}_{2} \mathrm{O}_{4}$ nanoparticles decorated MoS ${ }_{2}$-reduced graphene oxide nanocomposite for improved microwave absorption and shielding performance. RSC Adv. 2019, 9, 21881-21892. [CrossRef]

54. Zhang, K.; Zhang, Q.; Gao, X.; Chen, X.; Shi, J.; Wu, J. Ellipsoidal Fe 3 O $4 @$ C nanoparticles decorated fluffy structured graphene nanocomposites and their enhanced microwave absorption properties. J. Mater. Sci. Mater. Electron. 2018, 29, 6785-6796. [CrossRef]

55. Liu, L.; Bian, X.-M.; Hou, Z.-L.; Wang, C.-Y.; Li, Z.S.; Hu, H.D.; Qi, X.; Zhang, X. Electromagnetic response of magnetic graphene hybrid fillers and their evolutionary behaviors. J. Mater. Sci. Mater. Electron. 2016, 27, 2760-2772. [CrossRef]

56. Yin, P.; Deng, Y.; Zhang, L.; Wu, W.; Wang, J.; Feng, X.; Sun, X.; Li, H.; Tao, Y. One-step hydrothermal synthesis and enhanced microwave absorption properties of $\mathrm{Ni0}$. 5Co0. 5Fe2O4/graphene composites in low frequency band. Ceram. Int. 2018, 44, 20896-20905. [CrossRef]

57. Chen, Y.; Pötschke, P.; Pionteck, J.; Voit, B.; Qi, H. Multifunctional Cellulose/rGO/ $\mathrm{Fe}_{3} \mathrm{O}_{4}$ Composite Aerogels for Electromagnetic Interference Shielding. ACS Appl. Mater. Interfaces 2020, 12, 22088-22098. [CrossRef]

58. Fang, H.; Guo, H.; Hu, Y.; Ren, Y.; Hsu, P.-C.; Bai, S.-L. In-situ grown hollow $\mathrm{Fe}_{3} \mathrm{O}_{4}$ onto graphene foam nanocomposites with high EMI shielding effectiveness and thermal conductivity. Compos. Sci. Technol. 2020, 188, 107975. [CrossRef]

59. Yan, J.; Huang, Y.; Chen, X.; Wei, C. Conducting polymers-NiFe2O4 coated on reduced graphene oxide sheets as electromagnetic (EM) wave absorption materials. Synth. Met. 2016, 221, 291-298. [CrossRef]

60. Kumar, A.; Singh, A.K.; Tomar, M.; Gupta, V.; Kumar, P.; Singh, K. Electromagnetic interference shielding performance of lightweight $\mathrm{NiFe} 2 \mathrm{O} 4 / \mathrm{rGO}$ nanocomposite in X-band frequency range. Ceram. Int. 2020, 46, 15473-15481. [CrossRef]

61. Jiang, S.; Qian, K.; Yu, K.; Zhou, H.; Weng, Y.; Zhang, Z. Controllable synthesis and microwave absorption properties of Fe $\mathrm{O}_{4} @$ f-GNPs nanocomposites. Compos. Commun. 2020, 20, 100363. [CrossRef]

62. Liu, G.; Jiang, W.; Wang, Y.; Zhong, S.; Sun, D.; Liu, J.; Li, F. One-pot synthesis of Ag@ Fe3O4/reduced graphene oxide composite with excellent electromagnetic absorption properties. Ceram. Int. 2015, 41, 4982-4988. [CrossRef]

63. Zhang, H.; Jia, Z.; Feng, A.; Zhou, Z.; Chen, L.; Zhang, C.; Liu, X.; Wu, G. In situ deposition of pitaya-like $\mathrm{F}_{\mathrm{e}} 3 \mathrm{O}_{4} @ \mathrm{C}$ magnetic microspheres on reduced graphene oxide nanosheets for electromagnetic wave absorber. Compos. Part B Eng. 2020, $199,108261$. [CrossRef]

64. Fei, Y.; Liang, M.; Chen, Y.; Zou, H. Sandwich-like Magnetic Graphene Papers Prepared with $\mathrm{MOF}-\mathrm{Derived} \mathrm{Fe}_{3} \mathrm{O}_{4}-\mathrm{C}$ for Absorption-Dominated Electromagnetic Interference Shielding. Ind. Eng. Chem. Res. 2020, 59, 154-165. [CrossRef]

65. Jeon, S.; Kim, J.; Kim, K.H. Microwave absorption properties of graphene oxide capsulated carbonyl iron particles. Appl. Surf. Sci. 2019, 475, 1065-1069. [CrossRef]

66. Sharif, F.; Arjmand, M.; Moud, A.A.; Sundararaj, U.; Roberts, E.P. Segregated hybrid poly (methyl methacrylate)/graphene/ magnetite nanocomposites for electromagnetic interference shielding. Acs Appl. Mater. Interfaces 2017, 9, 14171-14179. [CrossRef] [PubMed]

67. Guo, T.; Li, C.; Wang, Y.; Wang, Y.; Yue, J.; Tang, X.-Z. A highly flexible and porous graphene-based hybrid film with superior mechanical strength for effective electromagnetic interference shielding. Appl. Phys. A 2020, 126, 1-8. [CrossRef]

68. Liang, C.; Hamidinejad, M.; Ma, L.; Wang, Z.; Park, C.B. Lightweight and flexible graphene/SiC-nanowires/poly (vinylidene fluoride) composites for electromagnetic interference shielding and thermal management. Carbon 2020, 156, 58-66. [CrossRef]

69. Sabira, K.; Jayakrishnan, M.; Saheeda, P.; Jayalekshmi, S. On the absorption dominated EMI shielding effects in free standing and flexible films of poly (vinylidene fluoride)/graphene nanocomposite. Eur. Polym. J. 2018, 99, 437-444. [CrossRef]

70. Hong, S.Y.; Kim, Y.C.; Wang, M.; Nam, J.-D.; Suhr, J. Anisotropic electromagnetic interference shielding properties of polymerbased composites with magnetically-responsive aligned Fe3O4 decorated reduced graphene oxide. Eur. Polym. J. 2020, 127, 109595. [CrossRef]

71. Zubair, K.; Shakir, M.F.; Afzal, A.; Rehan, Z.; Nawab, Y. Effect of Barium Hexaferrites and Thermally Reduced Graphene Oxide on EMI Shielding Properties in Polymer Composites. J. Supercond. Nov. Magn. 2020, 34, 201-210. [CrossRef]

72. Rao, B.B.; Yadav, P.; Aepuru, R.; Panda, H.; Ogale, S.; Kale, S. Single-layer graphene-assembled 3D porous carbon composites with PVA and $\mathrm{Fe}_{3} \mathrm{O}_{4}$ nano-fillers: An interface-mediated superior dielectric and EMI shielding performance. Phys. Chem. Chem. Phys. 2015, 17, 18353-18363. [PubMed]

73. Qi, Q.; Ma, L.; Zhao, B.; Wang, S.; Liu, X.; Lei, Y.; Park, C.B. An effective design strategy for the sandwich structure of PVDF/GNPNi-CNT composites with remarkable electromagnetic interference shielding effectiveness. ACS Appl. Mater. Interfaces 2020, 12, 36568-36577. [CrossRef] [PubMed]

74. Shakir, M.F.; Khan, A.N.; Khan, R.; Javed, S.; Tariq, A.; Azeem, M.; Riaz, A.; Shafqat, A.; Cheema, H.M.; Akram, M.A. EMI shielding properties of polymer blends with inclusion of graphene nano platelets. Results Phys. 2019, 14, 102365. [CrossRef]

75. Bera, R.; Das, A.K.; Maitra, A.; Paria, S.; Karan, S.K.; Khatua, B.B. Salt leached viable porous $\mathrm{Fe}_{3} \mathrm{O}_{4}$ decorated polyanilineSWCNH/PVDF composite spectacles as an admirable electromagnetic shielding efficiency in extended Ku-band region. Compos. Part B Eng. 2017, 129, 210-220. [CrossRef]

76. Bansala, T.; Joshi, M.; Mukhopadhyay, S.; Doong, R.-A.; Chaudhary, M. Electrically conducting graphene-based polyurethane nanocomposites for microwave shielding applications in the Ku band. J. Mater. Sci. 2017, 52, 1546-1560. [CrossRef] 
77. Jaiswal, R.; Agarwal, K.; Kumar, R.; Kumar, R.; Mukhopadhyay, K.; Prasad, N.E. EMI and microwave absorbing efficiency of polyaniline-functionalized reduced graphene oxide $/ \gamma-\mathrm{Fe}_{2} \mathrm{O}_{3}$ /epoxy nanocomposite. Soft Matter 2020, 16, 6643-6653. [CrossRef] [PubMed]

78. Sambyal, P.; Dhawan, S.; Gairola, P.; Chauhan, S.S.; Gairola, S. Synergistic effect of polypyrrole $/ \mathrm{BST} / \mathrm{RGO} / \mathrm{Fe}_{3} \mathrm{O}_{4}$ composite for enhanced microwave absorption and EMI shielding in X-Band. Curr. Appl. Phys. 2018, 18, 611-618. [CrossRef]

79. Dalal, J.; Lather, S.; Gupta, A.; Dahiya, S.; Maan, A.; Singh, K.; Dhawan, S.; Ohlan, A. EMI shielding properties of laminated graphene and $\mathrm{PbTiO} 3$ reinforced poly (3, 4-ethylenedioxythiophene) nanocomposites. Compos. Sci. Technol. 2018, 165, 222-230. [CrossRef]

80. Shukla, V. Role of spin disorder in magnetic and EMI shielding properties of $\mathrm{Fe}_{3} \mathrm{O}_{4} / \mathrm{C} / \mathrm{PPy}$ core/shell composites. J. Mater. Sci. 2020, 55, 2826-2835. [CrossRef]

81. Wang, Y.; Wu, X.; Zhang, W.; Luo, C.; Li, J.; Wang, Q. 3D heterostructure of graphene@ $\mathrm{Fe}_{3} \mathrm{O}_{4} @ \mathrm{WO}_{3} @$ PANI: Preparation and excellent microwave absorption performance. Synth. Met. 2017, 231, 7-14. [CrossRef]

82. Wang, Z.; Cheng, Z.; Xie, L.; Hou, X.; Fang, C. Flexible and lightweight Ti3C2Tx MXene/Fe $3 \mathrm{O}_{4} @$ PANI composite films for high-performance electromagnetic interference shielding. Ceram. Int. 2020. [CrossRef]

83. Liu, H.; Liang, C.; Chen, J.; Huang, Y.; Cheng, F.; Wen, F.; Xu, B.; Wang, B. Novel 3D network porous graphene nanoplatelets/Fe3O4/epoxy nanocomposites with enhanced electromagnetic interference shielding efficiency. Compos. Sci. Technol. 2019, 169, 103-109. [CrossRef]

84. Chen, Y.; Zhang, H.-B.; Huang, Y.; Jiang, Y.; Zheng, W.-G.; Yu, Z.-Z. Magnetic and electrically conductive epoxy/graphene/carbonyl iron nanocomposites for efficient electromagnetic interference shielding. Compos. Sci. Technol. 2015, 118, 178-185. [CrossRef]

85. Preeti, S.; Dhawan, S.K.; Singh, A.P.; Singh, K.; Ohlan, A. Nano-ferrite and Reduced Graphene Oxide Embedded in Polyaniline Matrix for EMI Shielding Applications. Composites 2016, 12, 16.

86. Gargama, H.; Thakur, A.; Chaturvedi, S. Polyvinylidene fluoride/nanocrystalline iron composite materials for EMI shielding and absorption applications. J. Alloys Compd. 2016, 654, 209-215. [CrossRef]

87. Zdrojek, M.; Bomba, J.; Łapińska, A.; Dużyńska, A.; Żerańska-Chudek, K.; Suszek, J.; Stobiński, L.; Taube, A.; Sypek, M.; Judek, J. Graphene-based plastic absorber for total sub-terahertz radiation shielding. Nanoscale 2018, 10, 13426-13431. [CrossRef] [PubMed]

88. Wang, L.; Zhu, J.; Yang, H.; Wang, F.; Qin, Y.; Zhao, T.; Zhang, P. Fabrication of hierarchical graphene@ Fe3O4@ SiO2@ polyaniline quaternary composite and its improved electrochemical performance. J. Alloys Compd. 2015, 634, 232-238. [CrossRef]

89. Hamidinejad, M.; Zhao, B.; Zandieh, A.; Moghimian, N.; Filleter, T.; Park, C.B. Enhanced electrical and electromagnetic interference shielding properties of polymer-graphene nanoplatelet composites fabricated via supercritical-fluid treatment and physical foaming. ACS Appl. Mater. Interfaces 2018, 10, 30752-30761. [CrossRef] [PubMed]

90. Lu, S.; Bai, Y.; Wang, J.; Chen, D.; Ma, K.; Meng, Q.; Liu, X. Flexible GnPs/EPDM with excellent thermal conductivity and electromagnetic interference shielding properties. Nano 2019, 14, 1950075. [CrossRef]

91. Liang, L.; Xu, P.; Wang, Y.; Shang, Y.; Ma, J.; Su, F.; Feng, Y.; He, C.; Wang, Y.; Liu, C. Flexible polyvinylidene fluoride film with alternating oriented graphene/Ni nanochains for electromagnetic interference shielding and thermal management. Chem. Eng. J. 2020, 395, 125209. [CrossRef]

92. Vovchenko, L.L.; Lozitsky, O.; Oliynyk, V.; Zagorodnii, V.; Len, T.; Matzui, L.; Milovanov, Y.S. Dielectric and microwave shielding properties of three-phase composites graphite nanoplatelets/carbonyl iron/epoxy resin. Appl. Nanosci. 2020, 10, 4781-4790. [CrossRef]

93. Zhao, J.; Lin, J.; Xiao, J.; Fan, H. Synthesis and electromagnetic, microwave absorbing properties of polyaniline/graphene oxide/Fe 3 O 4 nanocomposites. RSC Adv. 2015, 5, 19345-19352. [CrossRef]

94. Li, M.; Yang, K.; Zhu, W.; Shen, J.; Rollinson, J.; Hella, M.; Lian, J. Copper-Coated Reduced Graphene Oxide Fiber Mesh-Polymer Composite Films for Electromagnetic Interference Shielding. ACS Appl. Nano Mater. 2020, 3, 5565-5574. [CrossRef]

95. Wu, J.; Ye, Z.; Ge, H.; Chen, J.; Liu, W.; Liu, Z. Modified carbon fiber/magnetic graphene/epoxy composites with synergistic effect for electromagnetic interference shielding over broad frequency band. J. Colloid Interface Sci. 2017, 506, 217-226. [CrossRef] [PubMed]

96. Sawai, P.; Chattopadhaya, P.; Banerjee, S. Synthesized reduce Graphene Oxide (rGO) filled Polyetherimide based nanocomposites for EMI Shielding applications. Mater. Today: Proc. 2018, 5, 9989-9999. [CrossRef]

97. Khodiri, A.A.; Al-Ashry, M.Y.; El-Shamy, A.G. Novel hybrid nanocomposites based on polyvinyl alcohol/graphene/magnetite nanoparticles for high electromagnetic shielding performance. J. Alloys Compd. 2020, 847, 156430. [CrossRef]

98. Shahzad, F.; Lee, S.H.; Hong, S.M.; Koo, C.M. Segregated reduced graphene oxide polymer composite as a high performance electromagnetic interference shield. Res. Chem. Intermed. 2018, 44, 4707-4719. [CrossRef]

99. Joseph, J.; Koroth, A.K.; John, D.A.; Sidpara, A.M.; Paul, J. Highly filled multilayer thermoplastic/graphene conducting composite structures with high strength and thermal stability for electromagnetic interference shielding applications. J. Appl. Polym. Sci. 2019, 136, 47792. [CrossRef]

100. Yadav, R.S.; Kuřitka, I.; Vilčáková, J.; Machovský, M.; Škoda, D.; Urbánek, P.; Masař, M.; Gořalik, M.; Urbánek, M.; Kalina, L. Polypropylene nanocomposite filled with spinel ferrite $\mathrm{NiFe} 2 \mathrm{O} 4$ nanoparticles and in-situ thermally-reduced graphene oxide for electromagnetic interference shielding application. Nanomaterials 2019, 9, 621. [CrossRef] 
101. Li, S.; Li, W.; Nie, J.; Liu, D.; Sui, G. Synergistic effect of graphene nanoplate and carbonized loofah fiber on the electromagnetic shielding effectiveness of PEEK-based composites. Carbon 2019, 143, 154-161. [CrossRef]

102. Zuo, Y.; Su, X.; Li, X.; Yao, Z.; Yu, T.; Zhou, J.; Li, J.; Lu, J.; Ding, J. Multimaterial 3D-Printing of Graphene/ $\mathrm{Li}_{0.35} \mathrm{Zn}_{0.3} \mathrm{Fe}_{2.35} \mathrm{O}_{4}$ and graphene/carbonyl iron composites with superior microwave absorption properties and adjustable bandwidth. Carbon 2020, 167, 62-74. [CrossRef]

103. Sur, U.K. Graphene: A Rising Star on the Horizon of Materials Science. Int. J. Electrochem. 2012, 2012, 1-12. [CrossRef]

104. Hansora, D.; Shimpi, N.; Mishra, S. Graphite to graphene via graphene oxide: An overview on synthesis, properties, and applications. Jom 2015, 67, 2855-2868. [CrossRef]

105. Wohlford, T.; Schaaf, J.; Taylor, L.; Furtsch, T.; Khor, E.; St. Clair, A.; Seymour, R. Conductive Polymers. In Plenum, New York; Springer: Boston, MA, USA, 1981.

106. Yasufuku, S. Technical progress of EMI shielding materials in Japan. IEEE Electr. Insul. Mag. 1990, 6, 21-30. [CrossRef]

107. Chandra, R.J.; Shivamurthy, B.; Kulkarni, S.D.; Kumar, M.S. Hybrid polymer composites for EMI shielding application-A review. Mater. Res. Express 2019, 6, 082008. [CrossRef]

108. Luo, X.; Chung, D. Electromagnetic interference shielding using continuous carbon-fiber carbon-matrix and polymer-matrix composites. Compos. Part B Eng. 1999, 30, 227-231. [CrossRef]

109. Mannodi-Kanakkithodi, A.; Chandrasekaran, A.; Kim, C.; Huan, T.D.; Pilania, G.; Botu, V.; Ramprasad, R. Scoping the polymer genome: A roadmap for rational polymer dielectrics design and beyond. Mater. Today 2018, 21, 785-796. [CrossRef]

110. Sanchez-Lengeling, B.; Aspuru-Guzik, A. Inverse molecular design using machine learning: Generative models for matter engineering. Science 2018, 361, 360-365. [CrossRef] [PubMed]

111. Butler, K.T.; Davies, D.W.; Cartwright, H.; Isayev, O.; Walsh, A. Machine learning for molecular and materials science. Nature 2018, 559, 547-555. [CrossRef]

112. Hamidi, Y.K.; Berrado, A.; Altan, M.C. Machine learning applications in polymer composites. In Proceedings of the 35th International Conference Of The Polymer Processing Society (PPS-35), Cesme-Izmir, Turkey, 26-30 May 2019; AIP Publishing: Oslo, Norway, 2020; Volume 2205, p. 020031.

113. Wu, S.; Kondo, Y.; Kakimoto, M.-a.; Yang, B.; Yamada, H.; Kuwajima, I.; Lambard, G.; Hongo, K.; Xu, Y.; Shiomi, J. Machinelearning-assisted discovery of polymers with high thermal conductivity using a molecular design algorithm. NPJ Comput. Mater. 2019, 5, 1-11. [CrossRef]

114. Kim, C.; Batra, R.; Chen, L.; Tran, H.; Ramprasad, R. Polymer design using genetic algorithm and machine learning. Comput. Mater. Sci. 2021, 186, 110067. [CrossRef]

115. Venkatram, S.; Batra, R.; Chen, L.; Kim, C.; Shelton, M.; Ramprasad, R. Predicting crystallization tendency of polymers using multifidelity information fusion and machine learning. J. Phys. Chem. B 2020, 124, 6046-6054. [CrossRef] [PubMed]

116. Chen, L.; Kim, C.; Batra, R.; Lightstone, J.P.; Wu, C.; Li, Z.; Deshmukh, A.A.; Wang, Y.; Tran, H.D.; Vashishta, P. Frequencydependent dielectric constant prediction of polymers using machine learning. NPJ Comput. Mater. 2020, 6, 1-9. [CrossRef]

117. Sharma, V.; Wang, C.; Lorenzini, R.G.; Ma, R.; Zhu, Q.; Sinkovits, D.W.; Pilania, G.; Oganov, A.R.; Kumar, S.; Sotzing, G.A. Rational design of all organic polymer dielectrics. Nat. Commun. 2014, 5, 1-8. [CrossRef]

118. Huan, T.D.; Mannodi-Kanakkithodi, A.; Ramprasad, R. Accelerated materials property predictions and design using motif-based fingerprints. Phys. Rev. B 2015, 92, 014106. [CrossRef]

119. d'Avezac, M.; Luo, J.-W.; Chanier, T.; Zunger, A. Genetic-algorithm discovery of a direct-gap and optically allowed superstructure from indirect-gap Si and Ge semiconductors. Phys. Rev. Lett. 2012, 108, 027401. [CrossRef] [PubMed]

120. Mannodi-Kanakkithodi, A.; Pilania, G.; Huan, T.D.; Lookman, T.; Ramprasad, R. Machine learning strategy for accelerated design of polymer dielectrics. Sci. Rep. 2016, 6, 1-10. [CrossRef]

121. Zhou, T.; Song, Z.; Sundmacher, K. Big data creates new opportunities for materials research: A review on methods and applications of machine learning for materials design. Engineering 2019, 5, 1017-1026. [CrossRef]

122. Tian, H.; Zhao, Y.; Kang, L.; Shang, Y.; Liu, X. Electromagnetic Interference Diagnosis Based on HPLC Timing Sequence Topology and Machine Learning. In Proceedings of IOP Conference Series: Earth and Environmental Science; IOP: NewYork, NY, USA, 2019; Volume 375, p. 052015.

123. Eliardsson, P.; Stenumgaard, P. Artificial Intelligence for Automatic Classification of Unintentional Electromagnetic Interference in Air Traffic Control Communications. In Proceedings of the 2019 International Symposium on Electromagnetic Compatibility-EMC EUROPE, Barcelona, Spain, 2-6 September 2019; pp. 896-901.

124. Wei, X.; Shu, Y.; Hu, Z.; Zhong, Y.; Wang, Y. A Summary of Artificial Neural Networks on Electromagnetic Interference Diagnosis. In Proceedings of the 2019 International Conference on Microwave and Millimeter Wave Technology (ICMMT), Guangzhou, China, 19-22 May 2019; pp. 1-3.

125. Shu, Y.-F.; Wei, X.-C.; Fan, J.; Yang, R.; Yang, Y.-B. An equivalent dipole model hybrid with artificial neural network for electromagnetic interference prediction. IEEE Trans. Microw. Theory Tech. 2019, 67, 1790-1797. [CrossRef]

126. Hu, Z.-K.; Zhong, Y.-H.; Wei, X.-C.; Wang, Y.-W.; Shu, Y.-F. A Novel Electromagnetic Interference Source Reconstruction Method based on Artificial Neural Network. In Proceedings of the 2018 12th International Symposium on Antennas, Propagation and EM Theory (ISAPE), Hangzhou, China, 3-6 December 2018; pp. 1-4.

127. Yan, L.; Zhao, X.; Zhao, H.; Zhou, H.; Huang, K. Artificial neural network modeling of electromagnetic interference caused by nonlinear devices inside a metal enclosure. J. Electromagn. Waves Appl. 2015, 29, 992-1004. [CrossRef] 
128. Ellithy, K.; Al-Badi, A.; Al-Alawi, S. An artificial neural network model for predicting electromagnetic interference effects on gas pipelines built in power lines row. Eng. Intell. Syst. 2004, 12, 229-235.

129. Zhou, C.; Wang, Z.; Liu, T.; Zhao, S.; Liang, Z. Low dropout linear regulator's electromagnetic interference damage model based on BP neural network. High Volt. Eng. 2016, 42, 973-979.

130. Qishuang, Y.T.M.; Qingming, X. Prediction of electromagnetic interference based on neural network. J. Beijing Univ. Aeronaut. Astronaut. 2013, 5, 152-158.

131. Li, H.; Zhao, D.; Xu, S.; Wang, P.; Chen, J. High Dimensional Electromagnetic Interference Signal Clustering Based On SOM Neural Network. Electronics 2016, 20, 27-31. [CrossRef]

132. Masugi, M. Self-organizing neural network-based analysis of electrostatic discharge for electromagnetic interference assessment. IEICE Trans. Commun. 2003, 86, 1991-2000.

133. Micu, D.D.; Czumbil, L.; Christoforidis, G.; Simion, E. Neural networks applied in electromagnetic interference problems. Rev. Roum. Sci. Tech. Ser. Electrotech. Energetique 2012, 57, 162-171.

134. Micu, D.D.; Czumbil, L.; Christoforidis, G.; Ceclan, A. Layer recurrent neural network solution for an electromagnetic interference problem. IEEE Trans. Magn. 2011, 47, 1410-1413. [CrossRef]

135. Micu, D.D.; Czumbil, L.; Ceclan, A.; Darabant, L.; Stet, D.; Christoforidis, G. Electromagnetic interferences between HV power lines and metallic pipelines evaluated with neural network technique. In Proceedings of the 2009 10th International Conference on Electrical Power Quality and Utilisation, Lodz, Poland, 15-17 September 2009; pp. 1-5.

136. Micu, D.D.; Czumbil, L.; Ceclan, A.; Simion, E.; Stet, D.; Cîmpan, L. Neural Network Evaluation of Electromagnetic Interferences between HV Power Lines and Underground Metallic Pipelines. J. Electr. Electron. Eng. 2009, 1, 73.

137. Czumbil, L.; Micu, D.; Ceclan, A. Artificial Intelligence Techniques Applied to Electromagnetic Interference Problems. In Proceedings of the International Conference on Advancements of Medicine and Health Care through Technology, Paraná, Argentina, 29-31 October 2014; pp. 339-344.

138. Micu, D.D.; Czumbil, L.; Polycarpou, A.; Ceclan, A.; Cimpan, L. Analysis of electromagnetic interference problems proposed to be through an innovative Monte Carlo-Neural Network method. In Proceedings of the 7th Mediterranean Conference and Exhibition on Power Generation, Transmission, Distribution and Energy Conversion (MedPower 2010), Agia Napa, Cyprus, 7-10 November 2010; pp. 1-6.

139. Micu, D.D.; Czumbil, L.; Christoforidis, G.; Ceclan, A. Proposed Monte Carlo-Neural Network method for solving electromagnetic interference problems. In Proceedings of the 45th International Universities Power Engineering Conference UPEC2010, Cardiff, UK, 31 August-3 September 2010; pp. 1-6.

140. Mosavi, A.; Rabczuk, T.; Varkonyi-Koczy, A.R. Reviewing the novel machine learning tools for materials design. In International Conference on Global Research and Education; Springer: Cham, Switzerland, September 2017; pp. 50-58.

141. Mosavi, A.; Rabczuk, T. Learning and intelligent optimization for material design innovation. In International Conference on Learning and Intelligent Optimization; Springer: Cham, Switzerland, June 2017; pp. 358-363. 